\title{
DYNAMIC PROGRAMMING PRINCIPLE FOR STOCHASTIC RECURSIVE OPTIMAL CONTROL PROBLEM WITH DELAYED SYSTEMS*
}

\author{
Li CHEN $^{1}$ AND ZHEN Wu ${ }^{2}$
}

\begin{abstract}
In this paper, we study one kind of stochastic recursive optimal control problem for the systems described by stochastic differential equations with delay (SDDE). In our framework, not only the dynamics of the systems but also the recursive utility depend on the past path segment of the state process in a general form. We give the dynamic programming principle for this kind of optimal control problems and show that the value function is the viscosity solution of the corresponding infinite dimensional Hamilton-Jacobi-Bellman partial differential equation.
\end{abstract}

Mathematics Subject Classification. 49L20, 60H10, 93E20.

Received June 7, 2010. Revised May 6, 2011

Published online 16 January 2012.

\section{INTRODUCTION}

The classical stochastic control system is governed by a nonlinear stochastic differential equation (SDE). This kind of stochastic optimal control problems has been studied extensively, both by the dynamic programming approach and by the Pontryagin stochastic maximum principle. In our paper, we are concerned with the dynamic programming principle. There are many works concerning this subject. Such as Yong and Zhou [13] for the classical stochastic control system, and Peng [10,11], Wu and $\mathrm{Yu}$ [12] for the stochastic recursive case.

The research of many natural and social phenomena shows that the future development of many processes depends not only on their present state but also essentially on their previous history. Such processes can be described by the stochastic differential delayed equation (SDDE). Many examples can be found in Mohammed $[8,9]$. Whereas the dynamic programming principle can also be extended to stochastic control problems with delay (see e.g. [6]), most problems remain practically intractable because of the complex infinite-dimensional state space framework. In [7], Larssen and Risebro consider a class of optimal consumption problems with the stochastic delayed systems for some special cases, which shows that the financial application of this kind of dynamic programming principle.

Keywords and phrases. Stochastic differential equation with delay, recursive optimal control problem, dynamic programming principle, Hamilton-Jacobi-Bellman equation.

* This work is partly supported by the Natural Science Foundation of P.R. China (10921101) and Shandong Province (JQ200801 and 2008BS01024), the National Basic Research Program of P.R. China (973 Program, No. 2007CB814904) and the Science Fund for Distinguished Young Scholars of Shandong University (2009JQ004), the Fundamental Research Funds for the Central Universities (2010QS05).

1 Department of Mathematics, China University of Mining \& Technology, Beijing 100083, P.R. China. chenli@cumtb.edu.cn

2 School of Mathematics, Shandong University, Jinan 250100, P.R. China. wuzhen@sdu.edu.cn 
However, Duffien and Epstein [2] showed that the personal utility at time $t$ is not only a function of the instantaneous consumption rate, but also of the future utility (corresponding to the future consumption). Also, from [3], we know that this kind of recursive utility can be described by backward stochastic differential equation(BSDE), and the stochastic differential recursive utility is an extension of the standard additive one. So one of our goals in this paper is to obtain the dynamic programming principle for the delayed stochastic optimal control problem with the recursive utility.

For this, we study one kind of delayed stochastic recursive optimal control problem with the cost functional described by the solution of a BSDE. We prove that the celebrated dynamic programming principle for this kind of optimal control problem still holds. Because of the absence of Itô's formula for the function of the history state and the infinite-dimensional difficulty, it is not easy to obtain the Hamilton-Jacobi-Bellman (HJB) equation which the optimal value function satisfies. To overcome this difficulty, with the help of the theory of generator for the operator and the semigroup properties, we obtain the corresponding HJB equation and show that the value function of the recursive optimal control problem is the viscosity solution of HJB equation.

The paper is organized as follows. In Section 2, we present some notations which will be used throughout the paper, and we formulate the recursive optimal control problem with delay. In Section 3, we prove that the celebrated dynamic programming principle still holds under our framework. In Section 4, we give some properties of the optimal value function. We obtain the corresponding HJB equation and show that the optimal value function is the viscosity solution of the HJB equation in this section. Moreover, in some special case, we can get the uniqueness result of the viscosity solution.

\section{Notations And Formulation of the PRoblem}

Let $(\Omega, \mathcal{F}, P)$ be a probability space. Given $0 \leq T<\infty$ denoting a fixed terminal time. Let $0 \leq \delta<\infty$ be a fixed constant, and $[-\delta, 0]$ be the duration of the bounded delay of the systems considered in our paper. For the sake of simplicity, denote $C:=C\left([-\delta, 0] ; \mathbb{R}^{n}\right)$, the Banach space of continuous paths $\gamma:[-\delta, 0] \rightarrow \mathbb{R}^{n}$ with norm $\|\gamma\|_{C}:=\sup _{-\delta \leq s \leq 0}|\gamma(s)|$, where $|\cdot|$ is the Euclidean norm on $\mathbb{R}^{n}$. And let $n, d \geq 1$ be integer.

If $\varphi \in C\left([-\delta, T] ; \mathbb{R}^{n}\right)$ and $0 \leq t \leq T$, let $\varphi_{t}$ be defined by

$$
\varphi_{t}(\theta)=\varphi(t+\theta), \quad-\delta \leq \theta \leq 0 .
$$

We note that $\varphi_{t}$ is the segment of the path of $\varphi$ from $t-\delta$ to $t$.

Throughout the end, let $\{W(t)\}_{0 \leq t \leq T}$ be a $d$-dimensional Brownian motion on completed filtered probability space $\left(\Omega, \mathcal{F}, P ;\left\{\mathcal{F}_{t}\right\}_{t \geq 0}\right)$, where $\mathcal{F}_{t}$ is the natural filtration of $\{W(t)\}$ with $\mathcal{F}_{0}$ contains all $P$-null sets of $\mathcal{F}$.

We also use the following notations in our paper:

$$
\begin{aligned}
L^{2}(\Omega, \mathcal{F}, P) & =\left\{\xi \text { is an } \mathcal{F}_{T}-\text { measurable random variable s.t. } \mathbb{E}|\xi|^{2}<+\infty\right\}, \\
H_{\mathcal{F}}^{2}(0, T) & =\left\{\left(\varphi_{t}, 0 \leq t \leq T\right) \text { is an adapted process s.t. } \mathbb{E} \int_{0}^{T}\left|\varphi_{t}\right|^{2} \mathrm{~d} t<+\infty\right\}, \\
\mathcal{S}_{\mathcal{F}}^{2}(0, T) & =\left\{\left(\varphi_{t}, 0 \leq t \leq T\right) \text { is an adapted process s.t. } \mathbb{E}\left(\sup _{0 \leq t \leq T}\left|\varphi_{t}\right|^{2}\right)<+\infty\right\}, \\
L^{2}\left(\Omega, C ; \mathcal{F}_{t}\right) & =\left\{\varphi: \Omega \rightarrow C \text { is } \mathcal{F}_{t}-\text { measurable s.t. }\|\varphi\|_{L^{2}(\Omega, C)}^{2}:=\mathbb{E}\left[\|\varphi(\omega)\|_{C}^{2}\right]<\infty\right\} .
\end{aligned}
$$

Moreover, we denote by $(\cdot \mid \cdot)$ the inner product in $L^{2}\left(\Omega, C ; \mathcal{F}_{t}\right)$, and $\langle\cdot, \cdot\rangle$ the inner product in $\mathbb{R}^{n}$. Given $\varphi$ and $\phi$ in $C$, we have defined as follows,

$$
(\varphi \mid \phi)=\int_{-\delta}^{0}\langle\varphi(\theta), \phi(\theta)\rangle \mathrm{d} \theta, \text { and }\|\varphi\|_{2}=(\varphi \mid \varphi)^{\frac{1}{2}} .
$$


We introduce the admissible control set $\mathcal{U}_{a d}$ defined by

$$
\mathcal{U}_{a d}:=\left\{v(\cdot) \in H_{\mathcal{F}}^{2}(0, T) \mid v(\cdot) \text { takes value in } U \subset \mathbb{R}^{k}\right\} .
$$

Here $U$ is a compact subset of $\mathbb{R}^{k}$, an element of $\mathcal{U}_{a d}$ is called an admissible control.

For a given admissible control, we consider the following system of controlled stochastic functional differential equations with a bounded memory:

$$
\left\{\begin{array}{l}
\mathrm{d} X(t)=b\left(t, X_{t}, v(t)\right) \mathrm{d} t+\sigma\left(t, X_{t}, v(t)\right) \mathrm{d} W(t), t \in[s, T] \\
X_{s}=\varphi_{s}
\end{array}\right.
$$

Here $X_{s}$ and $\varphi_{s}$ are defined similarly as in (2.1), i.e., for any $0 \leq s \leq T, X_{s}=X(s+\theta), \varphi_{s}=\varphi(s+\theta)$, $-\delta \leq \theta \leq 0$, and $\varphi \in L^{2}\left(\Omega, C ; \mathcal{F}_{s}\right)$ is regarded as the initial path. The maps

$$
b:[0, T] \times C \times U \rightarrow \mathbb{R}^{n} ; \quad \sigma:[0, T] \times C \times U \rightarrow \mathbb{R}^{n \times d}
$$

satisfy the following assumptions:

(A1) $b$ and $\sigma$ are continuous in $t$;

(A2) for each integer $m \geq 1$, there is a constant $L_{m}>0$ (independent of $t$ ) such that

$$
|b(t, \phi, v)-b(t, \hat{\phi}, \hat{v})|+|\sigma(t, \phi, v)-\sigma(t, \hat{\phi}, \hat{v})| \leq L_{m}\left(\|\phi-\hat{\phi}\|_{C}+|v-\hat{v}|\right),
$$

for any $0 \leq t \leq T, \phi, \hat{\phi} \in C$ with $\|\phi\|_{C} \leq L_{m},\|\hat{\phi}\|_{C} \leq L_{m}$ and $v, \hat{v} \in U$;

(A3) there is a constant $K>0$ such that

$$
|b(t, \phi, v)|+|\sigma(t, \phi, v)| \leq K\left(1+\|\phi\|_{C}+|v|\right),
$$

for any $0 \leq t \leq T, \phi \in C, v \in U$.

Under the above assumptions, for any $v(\cdot) \in \mathcal{U}_{a d}$, the control system (2.2) with the aftereffect has a unique strong solution $\left\{X^{s, \varphi ; v}(t), 0 \leq s \leq t \leq T\right\}$, and also we have the following estimates by the existence and uniqueness theorem of SDDE in Mohammed [8,9]:

Proposition 2.1. For all $s \in[0, T], \varphi, \hat{\varphi} \in L^{2}\left(\Omega, C ; \mathcal{F}_{s}\right), v(\cdot), \hat{v}(\cdot) \in \mathcal{U}_{a d}$,

$$
\mathbb{E}^{\mathcal{F}_{s}}\left[\left\|X_{t}^{s, \varphi ; v}\right\|_{C}^{2}\right] \leq \Lambda\left(1+\|\varphi\|_{C}^{2}\right) .
$$

If we define the following mapping

$$
\begin{aligned}
T_{t}^{s}: L^{2}\left(\Omega, C ; \mathcal{F}_{s}\right) \times U & \rightarrow L^{2}\left(\Omega, C ; \mathcal{F}_{t}\right), \\
(\varphi, v) & \rightarrow X_{t}^{s, \varphi ; v},
\end{aligned}
$$

then we have

$$
\mathbb{E}^{\mathcal{F}_{s}}\left[\left\|T_{t}^{S}(\varphi, v)-T_{t}^{s}(\hat{\varphi}, \hat{v})\right\|_{C}^{2}\right] \leq \Lambda\left(\|\varphi-\hat{\varphi}\|_{C}^{2}+\mathbb{E}^{\mathcal{F}_{s}}\left[\int_{s}^{T}|v(t)-\hat{v}(t)|^{2} \mathrm{~d} t\right]\right) .
$$

Remark 2.2. From now on, let $\Lambda$ be a constant which can be changed line by line throughout our paper.

Now for any admissible control $v(\cdot) \in \mathcal{U}_{a d}, 0 \leq s \leq t \leq T$, we consider the following BSDE:

$$
Y^{s, \varphi ; v}(t)=\Phi\left(X_{T}^{s, \varphi ; v}\right)+\int_{t}^{T} f\left(r, X_{r}^{s, \varphi ; v}, Y^{s, \varphi ; v}(r), Z^{s, \varphi ; v}(r), v(r)\right) \mathrm{d} r-\int_{t}^{T} Z^{s, \varphi ; v}(r) \mathrm{d} W(r),
$$


where

$$
f:[0, T] \times C \times \mathbb{R} \times \mathbb{R}^{d} \times U \rightarrow \mathbb{R}, \Phi: C \rightarrow \mathbb{R}
$$

satisfy the following conditions:

(A4) $f$ is $\mathcal{F}_{t}$ measurable and it is continuous in $t$;

(A5) for some constant $L>0$, and for any $\phi, \hat{\phi} \in C, y, \hat{y} \in \mathbb{R}, z, \hat{z} \in \mathbb{R}^{d}, v, \hat{v} \in U$, a.s.

$$
|f(t, \phi, y, z, v)-f(t, \hat{\phi}, \hat{y}, \hat{z}, \hat{v})|+|\Phi(\phi)-\Phi(\hat{\phi})| \leq L\left(\|\phi-\hat{\phi}\|_{C}+|y-\hat{y}|+|z-\hat{z}|+|v-\hat{v}|\right),
$$

and $\forall(\phi, v) \in C \times U$, we have

$$
|f(t, \phi, 0,0, v)|+|\Phi(\phi)| \leq K\left(1+\|\phi\|_{C}\right) .
$$

Then from the result of the classical BSDE, we have that BSDE (2.5) has a unique solution triple $\left(Y^{s, \varphi ; v}, Z^{s, \varphi ; v}\right) \in \mathcal{S}_{\mathcal{F}}^{2}(0, T) \times H_{\mathcal{F}}^{2}(0, T)$. Moreover we get the estimates for the solution of $(2.5)$.

Proposition 2.3. (A1)-(A5) hold, we have

$$
\mathbb{E}^{\mathcal{F}_{s}}\left\{\sup _{s \leq t \leq T}\left|Y^{s, \varphi ; v}(t)\right|^{2}+\int_{s}^{T}\left|Z^{s, \varphi ; v}(t)\right|^{2} \mathrm{~d} t\right\} \leq \Lambda\left(1+\|\varphi\|_{C}^{2}\right)
$$

and

$$
\begin{aligned}
& \mathbb{E}^{\mathcal{F}_{s}}\left\{\sup _{s \leq t \leq T}\left|Y^{s, \varphi ; v}(t)-Y^{s, \hat{\varphi} ; \hat{v}}(t)\right|^{2}+\int_{s}^{T}\left|Z^{s, \varphi ; v}(t)-Z^{s, \hat{\varphi} ; \hat{v}}(t)\right|^{2} \mathrm{~d} t\right\} \\
\leq & \Lambda\|\varphi-\hat{\varphi}\|_{C}^{2}+\Lambda \mathbb{E}^{\mathcal{F}_{s}}\left\{\int_{s}^{T}|v(t)-\hat{v}(t)|^{2} \mathrm{~d} s\right\} .
\end{aligned}
$$

Given a control process $v(\cdot) \in \mathcal{U}_{a d}$, we introduce the associated cost functional

$$
J(s, \varphi ; v(\cdot)):=\left.Y^{s, \varphi ; v}(t)\right|_{t=s}, \quad(s, \varphi) \in[0, T] \times C,
$$

For each initial datum $(s, \varphi) \in[0, T] \times C$, the optimal control problem is to find $v(\cdot) \in \mathcal{U}_{a d}$ so as to maximize the objective function $J$.

Remark 2.4. The problem we formulated is one kind of stochastic recursive optimal control problem with delay. In the financial market, $X^{s, \varphi ; v}(t)$ can represent the wealth of the investor and $Y^{s, \varphi ; v}(t)$ represents the recursive utility cost functional, (see, for example, Ref. [3]).

\section{Dynamic PRogramming PRINCIPLE of THE PROBlem}

In this section, we will prove that the dynamic programming principle still holds for the above optimization problem.

We define the value function of the optimal control problem

$$
u(s, \varphi):=\operatorname{esssup}_{v(\cdot) \in \mathcal{U}_{a d}} J(s, \varphi ; v(\cdot)), \quad(s, \varphi) \in[0, T] \times C .
$$

For each $s>0$, we denote by $\left\{\mathcal{F}_{t}^{s}, s \leq t \leq T\right\}$ the natural filtration of the Brownian motion $\{W(t)-W(s), s \leq$ $t \leq T\}$ augmented by the P-null sets of $\mathcal{F}$. Also we introduce the following subspaces of $\mathcal{U}_{\text {ad }}$ :

$$
\begin{aligned}
& \mathcal{U}_{a d}^{s}:=\left\{v(\cdot) \in \mathcal{U}_{a d} \mid v(t) \text { is } \mathcal{F}_{t}^{s} \text { progressively measurable, } \forall s \leq t \leq T\right\}, \\
& \overline{\mathcal{U}}_{a d}^{s}:=\left\{v(t)=\sum_{j=1}^{N} v^{j}(t) 1_{A_{j}} \mid v^{j}(t) \in \mathcal{U}_{a d}^{s},\left\{A_{j}\right\}_{j=1}^{N} \text { is a partition of }\left(\Omega, \mathcal{F}_{s}\right), N=1,2, \cdots\right\} .
\end{aligned}
$$




\section{Lemma 3.1.}

$$
\begin{aligned}
& X_{t}^{s, \varphi ; \sum_{j=1}^{N} v^{j} 1_{A_{j}}}=\sum_{j=1}^{N} 1_{A_{j}} X_{t}^{s, \varphi ; v^{j}},
\end{aligned}
$$

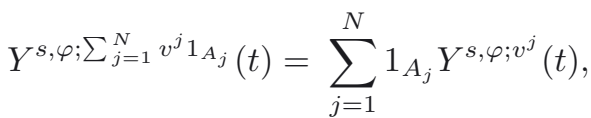

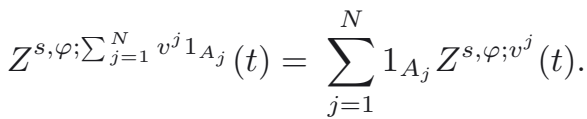

Proof. The main idea of the proof is essentially the same as that in Peng [11], hence we only give sketch for the different points. If $v=\sum_{j=1}^{N} 1_{A_{j}} v^{j}$, for each $j$ we denote

$$
\left(X^{j}(t), Y^{j}(t), Z^{j}(t)\right)=\left.\left(X^{s, \varphi ; v}(t), Y^{s, \varphi ; v}(t), Z^{s \varphi ; v}(t)\right)\right|_{v=v^{j}}, \text { and } X_{r}^{j}=\left.X_{r}\right|_{v=v^{j}} .
$$

We can see that $X^{j}(t), Y^{j}(t), Z^{j}(t)$ are the solutions of the following SDDE and BSDE respectively,

$$
\begin{aligned}
& X^{j}(t)=\varphi(s)+\int_{s}^{t} b\left(r, X_{r}^{j}, v^{j}(r)\right) \mathrm{d} r+\int_{s}^{t} \sigma\left(r, X_{r}^{j}, v^{j}(r)\right) \mathrm{d} W(r), t \in[s, T], \\
& Y^{j}(t)=\Phi\left(X_{T}^{j}\right)+\int_{t}^{T} f\left(r, X_{r}^{j}, Y^{j}(r), Z^{j}(r), v^{j}(r)\right) \mathrm{d} r-\int_{t}^{T} Z^{j}(r) \mathrm{d} W(r), t \in[s, T] .
\end{aligned}
$$

Multiplying $1_{A_{j}}$ on both sides of the above functions, we have

$$
\begin{aligned}
\sum_{j=1}^{N} 1_{A_{j}} X^{j}(t)= & \sum_{j=1}^{N} 1_{A_{j}} \varphi(s)+\int_{s}^{t} b\left(r, \sum_{j=1}^{N} 1_{A_{j}} X_{r}^{j}, \sum_{j=1}^{N} 1_{A_{j}} v^{j}(r)\right) \mathrm{d} r \\
& +\int_{s}^{t} \sigma\left(r, \sum_{j=1}^{N} 1_{A_{j}} X_{r}^{j}, \sum_{j=1}^{N} 1_{A_{j}} v^{j}(r)\right) \mathrm{d} W(r) \\
\sum_{j=1}^{N} 1_{A_{j}} Y^{j}(t)= & \Phi\left(\sum_{j=1}^{N} 1_{A_{j}} X_{T}^{j}\right)+\int_{t}^{T} f\left(r, \sum_{j=1}^{N} 1_{A_{j}} X_{r}^{j}, \sum_{j=1}^{N} 1_{A_{j}} Y^{j}(r), \sum_{j=1}^{N} 1_{A_{j}} Z^{j}(r)\right) \mathrm{d} r \\
& -\int_{t}^{T} \sum_{j=1}^{N} 1_{A_{j}} Z^{j}(r) \mathrm{d} W(r)
\end{aligned}
$$

by the virtue of $\sum_{j} \Phi\left(x_{j}\right) 1_{A_{j}}=\Phi\left(\sum_{j} x_{j} 1_{A_{j}}\right)$. Since $\sum_{j=1}^{N} 1_{A_{j}} \varphi=\varphi$ and the uniqueness of solutions for SDDE and BSDE, we have

$$
\begin{aligned}
& X^{s, \varphi ; v}(t)=X^{s, \varphi ; \sum_{j=1}^{N} 1_{A_{j}} v^{j}}(t)=\sum_{j=1}^{N} 1_{A_{j}} X^{j}(t), \\
& Y^{s, \varphi ; v}(t)=Y^{s, \varphi ; \sum_{j=1}^{N} 1_{A_{j}} v^{j}}(t)=\sum_{j=1}^{N} 1_{A_{j}} Y^{j}(t), \\
& Z^{s, \varphi ; v}(t)=Z^{s, \varphi ; \sum_{j=1}^{N} 1_{A_{j}} v^{j}}(t)=\sum_{j=1}^{N} 1_{A_{j}} Z^{j}(t) .
\end{aligned}
$$


And also we have

$$
X_{t}^{s, \varphi ; v}=X_{t}^{s, \varphi ; \sum_{j=1}^{N} 1_{A_{j}} v^{j}}=\sum_{j=1}^{N} 1_{A_{j}} X_{t}^{j} .
$$

Proposition 3.2. Under the assumptions $(A 1)-(A 5)$, the value function $u(s, \varphi)$ defined in $(3.1)$ is a deterministic function.

Proof. Firstly, we will prove that

$$
\operatorname{essup}_{v(\cdot) \in \mathcal{U}_{a d}} J(s, \varphi ; v(\cdot))=\operatorname{essup}_{v(\cdot) \in \overline{\mathcal{U}}_{a d}^{s}} J(s, \varphi ; v(\cdot)) .
$$

From the fact that $\overline{\mathcal{U}}_{a d}^{s}$ is a subset of $\mathcal{U}_{a d}$, we have

$$
\operatorname{essup}_{v(\cdot) \in \mathcal{U}_{a d}} J(s, \varphi ; v(\cdot)) \geq \operatorname{esssup}_{v(\cdot) \in \overline{\mathcal{U}}_{a d}^{s}} J(s, \varphi ; v(\cdot)) .
$$

Consequently, we only need to prove the inverse inequality. For any $v(\cdot), \hat{v}(\cdot) \in \mathcal{U}_{a d}$, we have

$$
\mathbb{E}\left\{\left|Y^{s, \varphi ; v}(s)-Y^{s, \varphi ; \hat{v}}(s)\right|\right\} \leq \Lambda \mathbb{E} \int_{s}^{T}|v(t)-\hat{v}(t)|^{2} \mathrm{~d} t
$$

by Proposition 2.3. Note that $\overline{\mathcal{U}}_{a d}^{s}$ is dense in $\mathcal{U}_{a d}$ under the norm of $H_{\mathcal{F}}^{2}(0, T)$, so we know that, for each $v(\cdot) \in \mathcal{U}_{a d}$, there exists a sequence $\left\{v_{n}(\cdot)\right\}_{n=1}^{\infty} \in \overline{\mathcal{U}}_{a d}^{s}$ such that

$$
\lim _{n \rightarrow \infty} \mathbb{E}\left\{\left|Y^{s, \varphi ; v_{n}}(s)-Y^{s, \varphi ; v}(s)\right|^{2}\right\}=0 .
$$

Thence, there exists a subsequence, we also denote $\left\{v_{n}(\cdot)\right\}_{n=1}^{\infty}$, such that

$$
\lim _{n \rightarrow \infty} Y^{s, \varphi ; v_{n}}(s)=Y^{s, \varphi ; v}(s), \text { a.s. }
$$

i.e.,

$$
\lim _{n \rightarrow \infty} J\left(s, \varphi ; v_{n}(\cdot)\right)=J(s, \varphi ; v(\cdot)), \text { a.s. }
$$

By the arbitrariness of $v(\cdot)$ and the definition of essential supremum, we get

$$
\operatorname{essup}_{v(\cdot) \in \overline{\mathcal{U}}_{a d}^{s}} J(s, \varphi ; v(\cdot)) \geq \operatorname{essup}_{v(\cdot) \in \mathcal{U}_{a d}} J(s, \varphi ; v(\cdot)) .
$$

Then (3.2) is proved.

Secondly, we want to prove

$$
\operatorname{essup}_{v(\cdot) \in \overline{\mathcal{U}}_{a d}^{s}} J(s, \varphi ; v(\cdot))=\operatorname{esssup}_{v(\cdot) \in \mathcal{U}_{a d}^{s}} J(s, \varphi ; v(\cdot)) .
$$

Obviously,

$$
\operatorname{essup}_{v(\cdot) \in \overline{\mathcal{U}}_{a d}^{s}} J(s, \varphi ; v(\cdot)) \geq \operatorname{esssup}_{v(\cdot) \in \mathcal{U}_{a d}^{s}} J(s, \varphi ; v(\cdot)) .
$$

On the other hand, from Lemma 3.1, we have

$$
J(s, \varphi ; v(\cdot))=J\left(s, \varphi ; \sum_{j=1}^{N} 1_{A_{j}} v^{j}(\cdot)\right)=\sum_{j=1}^{N} 1_{A_{j}} J\left(s, \varphi ; v^{j}(\cdot)\right)
$$

for all $v(\cdot) \in \overline{\mathcal{U}}_{a d}^{s}$. Note that $v^{j}(\cdot)(j=1,2, \ldots, N)$ are $\left\{\mathcal{F}_{t}^{s}\right\}$ progressively measurable, then $J\left(s, \varphi ; v^{j}(\cdot)\right)(j=$ $1,2, \ldots, N)$ are deterministic. Without loss of generality, we assume that

$$
J\left(s, \varphi ; v^{1}(\cdot)\right) \geq J\left(s, \varphi ; v^{j}(\cdot)\right), \quad \forall j=2,3, \ldots, N .
$$


Then

$$
J(s, \varphi ; v(\cdot)) \leq J\left(s, \varphi ; v^{1}(\cdot)\right) \leq \operatorname{esssup}_{v(\cdot) \in \mathcal{U}_{a d}^{s}} J(s, \varphi ; v(\cdot)) .
$$

Since $v(\cdot)$ is arbitrary, we obtain that

$$
\operatorname{essup}_{v(\cdot) \in \overline{\mathcal{U}}_{a d}^{s}} J(s, \varphi ; v(\cdot)) \leq \operatorname{esssup}_{v(\cdot) \in \mathcal{U}_{a d}^{s}} J(s, \varphi ; v(\cdot)) .
$$

The desired equality (3.3) is obtained.

From the definition of $\mathcal{U}_{a d}^{s}$, we know that the cost functional $J(s, \varphi ; v(\cdot))$ is deterministic when $v(\cdot) \in \mathcal{U}_{a d}^{s}$. Hence we get

$$
u(s, \varphi)=\sup _{v(\cdot) \in \mathcal{U}_{a d}^{s}} J(s, \varphi ; v(\cdot))
$$

is deterministic which is our desired result.

Next, we will explore the continuity of value function $u(s, \varphi)$ with respect to $\varphi$.

Lemma 3.3. For each $s \in[0, T]$, and $\varphi, \hat{\varphi} \in C$, we have

$$
\begin{aligned}
& \text { (i) }|u(s, \varphi)-u(s, \hat{\varphi})|^{2} \leq \Lambda\|\varphi-\hat{\varphi}\|_{C}^{2} ; \\
& \text { (ii) }|u(s, \varphi)| \leq \Lambda\left(1+\|\varphi\|_{C}\right) .
\end{aligned}
$$

Proof. From Proposition 2.3, for each admissible control $v(\cdot) \in \mathcal{U}_{a d}$, we have

$$
\begin{gathered}
|J(s, \varphi ; v(\cdot))| \leq \Lambda\left(1+\|\varphi\|_{C}\right), \\
|J(s, \varphi ; v(\cdot))-J(s, \hat{\varphi} ; v(\cdot))|^{2} \leq \Lambda\|\varphi-\hat{\varphi}\|_{C}^{2} .
\end{gathered}
$$

On the other hand, for each $\epsilon>0$, there exists a $v(\cdot), \hat{v}(\cdot) \in \mathcal{U}_{\text {ad }}$ s.t.

$$
J(s, \varphi ; \hat{v}(\cdot)) \leq u(s, \varphi) \leq J(s, \varphi ; v(\cdot))+\epsilon, J(s, \hat{\varphi} ; v(\cdot)) \leq u(s, \hat{\varphi}) \leq J(s, \hat{\varphi} ; \hat{v}(\cdot))+\epsilon .
$$

Then from (3.4), we get

$$
-\Lambda\left(1+\|\varphi\|_{C}\right) \leq J(s, \varphi ; \hat{v}(\cdot)) \leq u(s, \varphi) \leq J(s, \varphi ; v(\cdot))+\epsilon \leq \Lambda\left(1+\|\varphi\|_{C}\right)+\epsilon .
$$

Then (ii) comes from the arbitrariness of $\epsilon$. Similarly,

$$
\begin{aligned}
& J(s, \varphi ; \hat{v}(\cdot))-J(s, \hat{\varphi} ; \hat{v}(\cdot))-\epsilon \leq u(s, \varphi)-u(s, \hat{\varphi}) \leq J(s, \varphi ; v(\cdot))-J(s, \hat{\varphi} ; v(\cdot))+\epsilon, \\
& \quad|u(s, \varphi)-u(s, \hat{\varphi})| \\
& \leq \quad \max \{|J(s, \varphi ; v(\cdot))-J(s, \hat{\varphi} ; v(\cdot))|,|J(s, \varphi ; \hat{v}(\cdot))-J(s, \hat{\varphi} ; \hat{v}(\cdot))|\}+\epsilon, \\
& \quad|u(s, \varphi)-u(s, \hat{\varphi})|^{2} \\
& \leq 2 \max \left\{|J(s, \varphi ; v(\cdot))-J(s, \hat{\varphi} ; v(\cdot))|^{2},|J(s, \varphi ; \hat{v}(\cdot))-J(s, \hat{\varphi} ; \hat{v}(\cdot))|^{2}\right\}+2 \epsilon^{2} \\
& \leq 2 \Lambda\|\varphi-\hat{\varphi}\|_{C}^{2}+2 \epsilon^{2} .
\end{aligned}
$$

So $(i)$ holds. 
From (2.8), we know $Y^{s, \varphi ; v}(s)=J(s, \varphi ; v(\cdot))$ for deterministic $\varphi \in C$. About $\zeta \in L^{2}\left(\Omega, C ; \mathcal{F}_{s}\right)$, we need the following result:

Lemma 3.4. For any bounded $\zeta \in L^{2}\left(\Omega, C ; \mathcal{F}_{s}\right)$, we can find a sequence

$$
\eta_{m}=\sum_{j=1}^{m} 1_{A_{j}} \varphi^{j}
$$

converges to $\zeta$ in $L^{2}\left(\Omega, C ; \mathcal{F}_{s}\right)$, where $\varphi^{j}$ is a function defined on $[-\delta, 0]$ for $1 \leq j \leq m$ and $\left\{A_{j}\right\}_{j=1}^{m}$ is a partition of $\left(\Omega, \mathcal{F}_{s}\right)$.

Proof. For any bounded $\zeta \in L^{2}\left(\Omega, C ; \mathcal{F}_{s}\right)$, we also denote it by $\zeta(r, \omega), r \in[s-\delta, s]$. We define

$$
\zeta_{m}(r, \omega)=\sum_{i=-2^{m}}^{-1} \zeta\left(\frac{i}{2^{m}} \delta, \omega\right) 1_{\left[\frac{i}{2^{m}} \delta, \frac{i+1}{2^{m}} \delta\right)}(r), \quad-\delta \leq r \leq 0 .
$$

For every $\omega \in \Omega$, since the trajectory is continuous on a closed interval $[s-\delta, s]$, so the continuity is uniform. We have

$$
\lim _{m \rightarrow \infty}\left\|\zeta_{m}(\cdot, \omega)-\zeta(\cdot, \omega)\right\|_{C}=0, \quad \text { for any } \omega \in \Omega .
$$

Since $\zeta_{m}$ is uniformly bounded, by Lebesgue's dominated convergence theorem, we have

$$
\lim _{m \rightarrow \infty}\left\|\zeta_{m}-\zeta\right\|_{L^{2}(\Omega, C)}^{2}=\lim _{m \rightarrow \infty} \mathbb{E}\left[\left\|\zeta_{m}(\cdot, \omega)-\zeta(\cdot, \omega)\right\|_{C}^{2}\right]=0
$$

So, for any given $\epsilon>0$, there exists $M>0$ such that, whenever $m>M$, we have

$$
\left\|\zeta_{m}-\zeta\right\|_{L^{2}(\Omega, C)} \leq \frac{\epsilon}{3}
$$

For each $i$ and $m$, let us turn to analyze the $\mathcal{F}_{s}$-measurable random variable $\zeta\left(\frac{i}{2^{m}} \delta, \omega\right)$. It is easy to know that there exists a partition $\left\{A_{j}^{i, m}\right\}_{j=1}^{N_{i, m}}$ of $\left(\Omega, \mathcal{F}_{s}\right)$, and there exists a sequence of $\phi_{j}^{i, m} \in \mathbb{R}^{n}, j=1,2, \ldots, N_{i, m}$, such that

$$
\left|\sum_{j=1}^{N_{i, m}} 1_{A_{j}^{i, m}}(\omega) \phi_{j}^{i, m}-\zeta\left(\frac{i}{2^{m}} \delta, \omega\right)\right| \leq \frac{\epsilon}{3}, \quad \text { for any }(i, m) .
$$

We notice that the partitions above depend on $(i, m)$. Without loss of generality, we will use a thinner partition $\left\{A_{j}^{m}\right\}_{j=1}^{N_{m}}$ of $\left(\Omega, \mathcal{F}_{s}\right)$ to keep (3.8) to hold for all $i$, and the new partition is generated by the all partitions $\left\{A_{j}^{i, m}\right\}_{j=1}^{N_{i, m}}$. The advantage of the thinnest partition is that only depending on $m$. Then we have

$$
\left|\sum_{j=1}^{N_{m}} 1_{A_{j}^{m}}(\omega) \phi_{j}^{i, m}-\zeta\left(\frac{i}{2^{m}} \delta, \omega\right)\right| \leq \frac{\epsilon}{3}, \quad \text { for any }(i, m) .
$$

We define

$$
\begin{aligned}
\eta_{m}(r, \omega) & =\sum_{i=-2^{m}}^{-1} \sum_{j=1}^{N_{m}} \phi_{j}^{i, m} 1_{A_{j}^{m}}(\omega) 1_{\left[\frac{i}{2^{m}} \delta, \frac{i+1}{2^{m}} \delta\right)}(r) \\
& =\sum_{j=1}^{N_{m}} 1_{A_{j}^{m}}(\omega) \sum_{i=-2^{m}}^{-1} \phi_{j}^{i, m} 1_{\left[\frac{i}{2^{m}} \delta, \frac{i+1}{2^{m}} \delta\right)}(r) \\
& =\sum_{j=1}^{N_{m}} 1_{A_{j}^{m}}(\omega) \tilde{\varphi}_{j}^{m}(r) .
\end{aligned}
$$


And for any $m$, we calculate

$$
\begin{aligned}
\left|\eta_{m}(r, \omega)-\zeta_{m}(r, \omega)\right| & =\left|\sum_{i=-2^{m}}^{-1} \sum_{j=1}^{N_{m}} \phi_{j}^{i, m} 1_{A_{j}^{m}}(\omega) 1_{\left[\frac{i}{2^{m}} \delta, \frac{i+1}{2^{m}} \delta\right)}(r)-\sum_{i=-2^{m}}^{-1} \zeta\left(\frac{i}{2^{m}} \delta, \omega\right) 1_{\left[\frac{i}{2^{m}} \delta, \frac{i+1}{2^{m}} \delta\right)}(r)\right| \\
& \leq \sum_{i=-2^{m}}^{-1}\left|\sum_{j=1}^{N_{m}} \phi_{j}^{i, m} 1_{A_{j}^{m}}(\omega)-\zeta\left(\frac{i}{2^{m}} \delta, \omega\right)\right| 1_{\left[\frac{i}{2^{m}} \delta, \frac{i+1}{2^{m}} \delta\right)}(r) \\
& \leq \frac{\epsilon}{3} \sum_{i=-2^{m}}^{-1} 1_{\left[\frac{i}{2^{m}} \delta, \frac{i+1}{2^{m}} \delta\right)}(r) \\
& =\frac{\epsilon}{3}
\end{aligned}
$$

Moreover

$$
\left\|\eta_{m}-\zeta_{m}\right\|_{L^{2}(\Omega, C)} \leq \frac{\epsilon}{3}
$$

Combining (3.7) and (3.9), we obtain the conclusion: for any given $\epsilon>0$, there exists $M>0$ such that, whenever $m>M,\left\|\eta_{m}-\zeta\right\|_{L^{2}(\Omega, C)} \leq \epsilon$. We complete the proof.

Lemma 3.5. We have

$$
J(s, \zeta ; v(\cdot))=Y^{s, \zeta ; v}(s)
$$

for any $s \in[0, T], v(\cdot) \in \mathcal{U}_{a d}$ and for any $\zeta \in L^{2}\left(\Omega, C ; \mathcal{F}_{s}\right)$.

Proof. First, we consider a simple case that $\zeta=\sum_{j=1}^{N} 1_{A_{j}} \varphi^{j}$ with $\left\{A_{j}\right\}_{j=1}^{N}$ is a finite partition of $\left(\Omega, \mathcal{F}_{s}\right)$, and $\varphi^{j} \in C[-\delta, 0]^{n}$, for $1 \leq j \leq N$. The similar argument in Lemma 3.1, we deduce that

$$
Y^{s, \zeta ; v}(s)=\sum_{j=1}^{N} 1_{A_{j}} Y^{s, \varphi^{j} ; v}(s)=\sum_{j=1}^{N} 1_{A_{j}} J\left(s, \varphi^{j} ; v(\cdot)\right)=J\left(s, \sum_{j=1}^{N} 1_{A_{j}} \varphi^{j} ; v(\cdot)\right)=J(s, \zeta ; v(\cdot)) .
$$

That implies (3.10) holds for the above simple case.

Next, given a bounded $\zeta \in L^{2}\left(\Omega, C ; \mathcal{F}_{s}\right)$, we can choose a sequence of simple process $\left\{\zeta_{j}\right\}$ similar to the form of $(3.6)$ such that $\left\{\zeta_{j}\right\}$ converges to $\zeta$ in $L^{2}\left(\Omega, C ; \mathcal{F}_{s}\right)$. Then, from Proposition 2.3 we can derive

$$
\mathbb{E}\left[\left|Y^{s, \zeta ; v}(s)-Y^{s, \zeta_{j} ; v}(s)\right|^{2}\right] \leq \Lambda\left\|\zeta-\zeta_{j}\right\|_{L^{2}(\Omega, C)} \rightarrow 0, \text { as } j \rightarrow \infty .
$$

At the same time, form (3.5) we have the following result about $J$

$$
\mathbb{E}\left[\left|J(s, \zeta ; v(\cdot))-J\left(s, \zeta_{j} ; v(\cdot)\right)\right|^{2}\right] \leq \Lambda\left\|\zeta-\zeta_{j}\right\|_{L^{2}(\Omega, C)} \rightarrow 0, \text { as } j \rightarrow \infty .
$$

As a result of $Y^{s, \zeta_{j} ; v}(s)=J\left(s, \zeta_{j} ; v(\cdot)\right)$, we get our desired conclusion. If $\zeta$ is unbounded, we can define $\zeta^{n}=$ $(\zeta \wedge n) \bigvee(-n)$, then $\zeta^{n}$ is bounded and

$$
\mathbb{E}\left[\left|Y^{s, \zeta ; v}(s)-Y^{s, \zeta^{n} ; v}(s)\right|^{2}\right] \leq \Lambda\left\|\zeta-\zeta^{n}\right\|_{L^{2}(\Omega, C)} \rightarrow 0, \text { as } n \rightarrow \infty .
$$

Repeat the processes similarly as the bounded case, and we can also get our desired results. 
In order to prove the dynamic programming principle, we need the following lemma:

Lemma 3.6. For each $v(\cdot) \in \mathcal{U}_{\text {ad }}$, fixed $s \in[0, T)$ and $\zeta \in L^{2}\left(\Omega, C ; \mathcal{F}_{s}\right)$, we have

$$
u(s, \zeta) \geq Y^{s, \zeta ; v}(s) .
$$

On the other hand, for each $\epsilon>0$, there is an admissible control $v(\cdot) \in \mathcal{U}_{\text {ad }}$, such that

$$
u(s, \zeta) \leq Y^{s, \zeta ; v}(s)+\epsilon, \text { a.s. }
$$

Proof. Same as that in the proof of Lemma 3.5, we first consider the case: $\zeta=\sum_{j=1}^{N} 1_{A_{j}} \varphi^{j}$. Then, for each $v(\cdot) \in \mathcal{U}_{a d}$, we have

$$
Y^{s, \zeta ; v}(s)=Y^{s, \sum_{j=1}^{N} 1_{A_{j}} \varphi^{j} ; v}(s)=\sum_{j=1}^{N} 1_{A_{j}} Y^{s, \varphi^{j} ; v}(s) \leq \sum_{j=1}^{N} 1_{A_{j}} u\left(s, \varphi^{j}\right)=u(s, \zeta) .
$$

Furthermore, if $\zeta \in L^{2}\left(\Omega, C ; \mathcal{F}_{s}\right)$, we can also choose a sequence of simple process $\left\{\zeta_{j}\right\}$ which converges to $\zeta$ in $L^{2}\left(\Omega, C ; \mathcal{F}_{s}\right)$. So,

$$
\mathbb{E}\left[\left|Y^{s, \zeta ; v}(s)-Y^{s, \zeta_{j} ; v}(s)\right|^{2}\right] \rightarrow 0 ; \quad \mathbb{E}\left[\left|u(s, \zeta)-u\left(s, \zeta_{j}\right)\right|^{2}\right] \rightarrow 0,(j \rightarrow \infty) .
$$

Then there exists a subsequence which we also denote it by $\left\{\zeta_{j}\right\}$ such that

$$
\lim _{j \rightarrow \infty} Y^{s, \zeta_{j} ; v}(s)=Y^{s, \zeta ; v}(s), \text { a.s., } \quad \lim _{j \rightarrow \infty} u\left(s, \zeta_{j}\right)=u(s, \zeta), \text { a.s. }
$$

With the help of $Y^{s, \zeta_{j} ; v}(s) \leq u\left(s, \zeta_{j}\right), j=1,2, \ldots$, we get (3.11).

We can use the similar method to prove (3.12). We first consider that $\zeta \in L^{\infty}\left(\Omega, C ; \mathcal{F}_{s}\right)$ with $\|\zeta\|_{C} \leq M$, then we can construct a random variable $\eta \in L^{\infty}\left(\Omega, C ; \mathcal{F}_{s}\right)$ with the form $\eta=\sum_{j=1}^{N} 1_{A_{j}} \varphi^{j}, \varphi^{j} \in C,\left\{A_{j}\right\}_{j=1}^{N}$ is also the partition of $\left(\Omega, \mathcal{F}_{s}\right)$ such that

$$
\|\zeta-\eta\|_{L^{2}(\Omega, C)} \leq \frac{\epsilon}{3 \Lambda}
$$

For any $v(\cdot) \in \mathcal{U}_{a d}$, we have

$$
\left|Y^{s, \zeta ; v}(s)-Y^{s, \eta ; v}(s)\right| \leq \frac{\epsilon}{3},|u(s, \zeta)-u(s, \eta)| \leq \frac{\epsilon}{3} .
$$

Then, For each $\varphi^{j}$, we can choose an $\mathcal{F}_{t}^{s}$-adapted admissible control $v^{j}(\cdot)$ such that

$$
u\left(s, \varphi^{j}\right) \leq Y^{s, \varphi^{j} ; v^{j}}(s)+\frac{\epsilon}{3} .
$$

If we set $v(\cdot)=\sum_{j=1}^{N} 1_{A_{j}} v^{j}(\cdot)$, we can derive the following result with (3.13):

$$
\begin{aligned}
Y^{s, \zeta ; v}(s) & \geq-\left|Y^{s, \eta ; v}(s)-Y^{s, \zeta, v}(s)\right|+Y^{s, \eta ; v}(s) \geq-\frac{\epsilon}{3}+\sum_{j=1}^{N} 1_{A_{j}} Y^{s, \varphi^{j} ; v^{j}}(s) \\
& \geq-\frac{\epsilon}{3}+\sum_{j=1}^{N} 1_{A_{j}}\left(u\left(s, \varphi^{j}\right)-\frac{\epsilon}{3}\right)=-\frac{2}{3} \epsilon+\sum_{j=1}^{N} 1_{A_{j}} u\left(s, \varphi^{j}\right) \\
& =-\frac{2}{3} \epsilon+u(s, \eta) \geq-\epsilon+u(s, \zeta) .
\end{aligned}
$$

That is to say that $(3.12)$ holds for any $\zeta \in L^{\infty}\left(\Omega, C ; \mathcal{F}_{s}\right)$. 
For the general case $\zeta \in L^{2}\left(\Omega, C ; \mathcal{F}_{s}\right)$, fortunately we know that $\zeta$ can be decomposed as

$$
\zeta=\sum_{j=1}^{\infty} 1_{A_{j}} \zeta_{j}
$$

where $\left\{A_{j}\right\}_{j=1}^{\infty}$ is also a partition of $\left(\Omega, \mathcal{F}_{s}\right)$ and $\zeta_{j} \in L^{\infty}\left(\Omega, C ; \mathcal{F}_{s}\right)$ with $\left\|\zeta_{j}\right\|_{C} \leq j, j=1,2, \cdots$. Thence, for each $\zeta_{j}$, there exists $v^{j}(\cdot) \in \mathcal{U}_{a d}$ such that

$$
u\left(s, \zeta_{j}\right) \leq Y^{s, \zeta_{j} ; v^{j}}(s)+\epsilon .
$$

Denoting $v(\cdot)=\sum_{j=1}^{\infty} 1_{A_{j}} v^{j}(\cdot)$, we get

$$
\begin{aligned}
u(s, \zeta) & =u\left(s, \sum_{j=1}^{\infty} 1_{A_{j}} \zeta_{j}\right)=\sum_{j=1}^{\infty} 1_{A_{j}} u\left(s, \zeta_{j}\right) \leq \sum_{j=1}^{\infty} 1_{A_{j}}\left(Y^{s, \zeta_{j} ; v^{j}}(s)+\epsilon\right) \\
& =\sum_{j=1}^{\infty} 1_{A_{j}} Y^{s, \zeta_{j} ; v^{j}}(s)+\epsilon=Y^{s, \zeta ; v}(s)+\epsilon .
\end{aligned}
$$

Then we complete our proof.

Next, we will introduce a family of backward semigroups which is embedded in [11].

Given the initial condition $(s, \varphi) \in[0, T) \times C$, and an admissible control $v(\cdot) \in \mathcal{U}_{a d}$. If $\tau \leq T-s$ is a positive number, and $\zeta \in L^{2}\left(\Omega, C ; \mathcal{F}_{s+\tau}\right)$, we denote

$$
G_{s, s+\tau}^{s, \varphi ; v}[\zeta]:=Y^{s, \varphi ; v}(s),
$$

where $\left(Y^{s, \varphi ; v}(t), Z^{s, \varphi ; v}(t)_{s \leq t \leq s+\tau}\right.$ is the solution of the following BSDE

$$
\left\{\begin{aligned}
-\mathrm{d} Y^{s, \varphi ; v}(t) & =f\left(t, X_{t}^{s, \varphi ; v}, Y^{s, \varphi ; v}(t), Z^{s, \varphi ; v}(t), v(t)\right) \mathrm{d} t-Z^{s, \varphi ; v}(t) \mathrm{d} W(t), \quad t \in[s, s+\tau], \\
Y^{s, \varphi ; v}(s+\tau) & =\zeta .
\end{aligned}\right.
$$

Obviously,

$$
G_{s, T}^{s, \varphi ; v}\left[\Phi\left(X_{T}^{s, \varphi ; v}\right)\right]=G_{s, s+\tau}^{s, \varphi ; v}\left[Y^{s, \varphi ; v}(s+\tau)\right] .
$$

Then we can get the main result of this section, the generalized dynamic programming principle for our stochastic recursive optimal control problem with delayed system.

Theorem 3.7 (the generalized dynamic programming principle). Let (A1)-(A5) hold. Then the value function $u(s, \varphi)$ defined by (3.1) for our optimal control problem with the delayed system has the following property: for each $0 \leq \tau \leq T-s$,

$$
u(s, \varphi)=\operatorname{essup}_{v(\cdot) \in \mathcal{U}_{a d}} G_{s, s+\tau}^{s, \varphi ; v}\left[u\left(s+\tau, X_{s+\tau}^{s, \varphi ; v}\right)\right] .
$$

Proof. We have

$$
\begin{aligned}
u(s, \varphi) & =\operatorname{essup}_{v(\cdot) \in \mathcal{U}_{a d}} G_{s, T}^{s, \varphi ; v}\left[\Phi\left(X_{T}^{s, \varphi ; v}\right)\right]=\operatorname{esssup}_{v(\cdot) \in \mathcal{U}_{a d}} G_{s, s+\tau}^{s, \varphi ; v}\left[Y^{s, \varphi ; v}(s+\tau)\right] \\
& =\operatorname{essup}_{v(\cdot) \in \mathcal{U}_{a d}} G_{s, s+\tau}^{s, \varphi ; v}\left[Y^{s+\tau, X_{t+\tau}^{s, \varphi ; v} ; v}(s+\tau)\right] .
\end{aligned}
$$

From Lemma 3.6 and the comparision theorem of BSDE, we have

$$
u(s, \varphi) \leq \operatorname{essup}_{v(\cdot) \in \mathcal{U}_{a d}} G_{s, s+\tau}^{s, \varphi ; v}\left[u\left(s+\tau, X_{s+\tau}^{s, \varphi ; v}\right)\right] .
$$


Moreover, also by Lemma 3.6, we can conclude that: for every $\epsilon>0$, we can find an admissible control $\hat{v}(\cdot) \in \mathcal{U}_{a d}$ such that

$$
u\left(s+\tau, X_{s+\tau}^{s, \varphi ; v}\right) \leq Y^{s+\tau, X_{s+\tau}^{s, \varphi ; v} ; \hat{v}}(s+\tau)+\epsilon .
$$

For each $v(\cdot) \in \mathcal{U}_{a d}$, we denote $\bar{v}(t)=1_{\{t \leq s+\tau\}} v(t)+1_{\{t>s+\tau\}} \hat{v}(t)$. From the above inequality and the comparison theorem, we get

$$
Y^{s+\tau, X_{s+\tau}^{s, \varphi ; \bar{v}} ; \bar{v}}(s+\tau) \geq u\left(s+\tau, X_{s+\tau}^{s, \varphi ; \bar{v}}\right)-\epsilon, \quad u(s, \varphi) \geq \operatorname{essup}_{\bar{v}(\cdot) \in \mathcal{U}_{a d}^{\hat{v}}} G_{s, s+\tau}^{s, \varphi ; \bar{v}}\left[u\left(s+\tau, X_{s+\tau}^{s, \varphi ; \bar{v}}\right)-\epsilon\right],
$$

and here

$$
\mathcal{U}_{a d}^{\hat{v}}:=\left\{\bar{v}(\cdot) \in \mathcal{U}_{a d} \mid \bar{v}(t)=1_{\{t \leq s+\tau\}} v(t)+1_{\{t>s+\tau\}} \hat{v}(t) \text { for some } v(\cdot) \in \mathcal{U}_{a d}\right\} .
$$

From the estimates of BSDE, there exists a positive constant $\Lambda_{0}$ such that

$$
u(s, \varphi) \geq \operatorname{esssup}_{\bar{v}(\cdot) \in \mathcal{U}_{a d}^{\hat{v}}} G_{s, s+\tau}^{s, \varphi ; \bar{v}}\left[u\left(s+\tau, X_{s+\tau}^{s, \varphi ; \bar{v}}\right)\right]-\Lambda_{0} \epsilon .
$$

Letting $\epsilon \downarrow 0$, we obtain

$$
u(s, \varphi) \geq \operatorname{essup}_{\bar{v}(\cdot) \in \mathcal{U}_{a d}^{\hat{v}}} G_{s, s+\tau}^{s, \varphi ; \bar{v}}\left[u\left(s+\tau, X_{s+\tau}^{s, \varphi ; \bar{v}}\right)\right] .
$$

From the definition of $\bar{v}(\cdot)$ and the arbitrariness of $v(\cdot) \in \mathcal{U}_{a d}$, we know

$$
u(s, \varphi) \geq \operatorname{essup}_{v(\cdot) \in \mathcal{U}_{a d}} G_{s, s+\tau}^{s, \varphi}\left[u\left(s+\tau, X_{s+\tau}^{s, \varphi ; v}\right)\right] .
$$

Then (3.15) is obtained.

Remark 3.8. (3.15) implies that the value function $u(s, \varphi)$ obeys the dynamic programming principle which holds also for the usual optimization case without the recursive utility (see Refs. [6,7]).

\section{The Hamilton-Jacobi-Bellman equations}

We call (3.15) the dynamic programming equation. It seems impossible to solve such an equation directly. In this section, we will prove that, under some smooth conditions, the value function $u(\cdot, \cdot)$ defined by $(3.1)$ satisfies a kind of partial differential equations-HJB equations.

\subsection{The notions and definitions}

We first introduce some notations and definitions which also used in $[1,4,5,9]$.

Let $C_{b}$ be the Banach space of all bounded uniformly continuous functions $\Phi: C \rightarrow \mathbb{R}$ with the sup norm

$$
\|\Phi\|_{C_{b}}:=\sup _{\eta \in C}|\Phi(\eta)|, \Phi \in C_{b} .
$$

Define the operator $P_{t}: C_{b} \rightarrow C_{b}, t \geq 0$, on $C_{b}$ by

$$
P_{t}(\Phi)(\eta):=\mathbb{E}\left[\Phi\left(X_{t}^{\eta}\right)\right], \quad t \geq 0, \quad \Phi \in C_{b}, \quad \eta \in C .
$$

For any $\Phi \in C_{b}$ and any finite Borel measure $\mu$ on $C$, define the pairing

$$
\langle\Phi, \mu\rangle:=\int_{\eta \in C} \Phi(\eta) \mathrm{d} \mu(\eta) .
$$

We also define a generator $\mathcal{A}: D(\mathcal{A}) \subset C_{b} \rightarrow C_{b}$ of $\left\{P_{t}\right\}_{t \geq 0}$ by the weak limit

$$
\mathcal{A}(\Phi)(\eta):=w-\lim _{t \rightarrow 0+} \frac{P_{t}(\Phi)-\Phi}{t},
$$

here $\Phi$ belongs to the domain $D(\mathcal{A})$ of $\mathcal{A}$ if and only if the above weak limit exists in $C_{b}$. 
Then we can easily obtain:

\section{Lemma 4.1.}

$$
\frac{\mathrm{d}}{\mathrm{d} t} P_{t}(\Phi)=\mathcal{A}\left(P_{t}(\Phi)\right)=P_{t}(\mathcal{A}(\Phi)), t \geq 0
$$

for any $\Phi \in D(\mathcal{A})$.

Let $F_{n}:=\left\{\kappa \mathbf{1}_{\{0\}}: \kappa \in \mathbb{R}^{n}\right\}$ and $C \oplus F_{n}:=\left\{\eta+\kappa \mathbf{1}_{\{0\}}: \eta \in C, \kappa \in \mathbb{R}^{n}\right\}$ with norm $\left\|\eta+\kappa \mathbf{1}_{\{0\}}\right\|:=\|\eta\|_{C}+|\kappa|$ for $\eta \in C, \kappa \in \mathbb{R}^{n}$, where $\mathbf{1}_{\{0\}}:[-\delta, 0] \rightarrow \mathbb{R}$ is defined by

$$
\mathbf{1}_{\{0\}}(\theta)=\left\{\begin{array}{l}
0, \text { for } \theta \in[-\delta, 0), \\
1, \text { for } \theta=0
\end{array}\right.
$$

For a Borel measurable function $\Phi: C \rightarrow \mathbb{R}$, we also define

$$
\mathcal{S}(\Phi)(\eta):=\lim _{t \rightarrow 0} \frac{\Phi\left(\tilde{\eta}_{t}\right)-\Phi(\eta)}{t}
$$

for all $\eta \in C$, where $\tilde{\eta}:[-\delta, T] \rightarrow \mathbb{R}^{n}$ is an extension of $\eta$ defined by

$$
\tilde{\eta}(t):=\left\{\begin{array}{l}
\eta(t), t \in[-\delta, 0), \\
\eta(0), t \geq 0,
\end{array}\right.
$$

and $\tilde{\eta}_{t} \in C$ is defined by $\tilde{\eta}_{t}(\theta):=\tilde{\eta}(t+\theta), \theta \in[-\delta, 0]$. Let $\hat{D}(\mathcal{S})$, the domain of $\mathcal{S}$, be the set of $\Phi: C \rightarrow \mathbb{R}$ such that the above limit exists for each $\eta \in C$. Define $D(\mathcal{S})$ as the set of all functions $\Phi:[0, T] \times C \rightarrow \mathbb{R}$ such that $\Phi(t, \cdot) \in \hat{D}(\mathcal{S}), \forall t \in[0, T]$.

In addition, for each sufficiently smooth function $\Phi$, we will denote its first and second Fréchet derivative with respect to $\eta \in C$ by $D \Phi$ and $D^{2} \Phi$. And let $C_{l i p}^{1,2}([0, T] \times C)$ be the set of functions $\Phi:[0, T] \times C \rightarrow \mathbb{R}$ such that $\frac{\partial \Phi}{\partial t}, D \Phi$ and $D^{2} \Phi$ exist and they are globally bounded and Lipschitz.

Then we can derive a formula for the generator $\mathcal{A}$.

Theorem $4.2\left(([9])\right.$. Suppose that $\Phi \in C_{\text {lip }}^{1,2}([0, T] \times C) \cap D(\mathcal{S})$. Let $v(\cdot) \in \mathcal{U}_{\text {ad }}$, and $\{X(t), t \in[s, T]\}$ be the $C$-valued Markov solution process of equation (2.2) with the initial data $\left(s, \eta_{s}\right) \in[0, T] \times C$. Then for $\Phi \in D(\mathcal{A})$,

$$
\begin{aligned}
\mathcal{A}(\Phi)\left(X_{t}\right)= & \frac{\partial}{\partial t} \Phi\left(t, X_{t}\right)+\mathcal{S}(\Phi)\left(X_{t}\right)+\overline{D \Phi\left(t, X_{t}\right)}\left(b\left(t, X_{t}, v(t)\right) \mathbf{1}_{\{\mathbf{0}\}}\right) \\
& +\frac{1}{2} \sum_{i=1}^{d} \overline{D^{2} \Phi\left(t, X_{t}\right)}\left(\sigma\left(t, X_{t}, v(t)\right) \mathbf{e}_{i} \mathbf{1}_{\{0\}}, \sigma\left(t, X_{t}, v(t)\right) \mathbf{e}_{i} \mathbf{1}_{\{0\}}\right),
\end{aligned}
$$

where $\overline{D \Phi}: C \oplus F_{n} \rightarrow \mathbb{R}, \overline{D^{2} \Phi}:\left(C \oplus F_{n}\right) \times\left(C \oplus F_{n}\right) \rightarrow \mathbb{R}$ are the continuous linear and bilinear extensions of $D \Phi, D^{2} \Phi$ respectively, and $\left\{\mathbf{e}_{i}\right\}_{i=1}^{d}$ is the standard basis in $\mathbb{R}^{d}$.

\subsection{Some properties of the value function}

We will explore some more properties of the value function defined in previous subsection.

Lemma 4.3. Under the assumptions $(A 1)-(A 5)$, for any $(s, \zeta) \in[0, T) \times L^{2}\left(\Omega, C ; \mathcal{F}_{s}\right)$ and $v \in \mathcal{U}_{\text {ad }}$, we have

$$
\lim _{t \rightarrow s^{+}}\left\|X_{t}^{s, \zeta ; v}-\zeta\right\|_{L^{2}(\Omega, C)}=0 .
$$

Proposition 4.4. Suppose $(A 1)-(A 5)$ hold, then the value function $u(s, \varphi)$ is continuous in $(s, \varphi) \in[0, T] \times C$. 
Proof. By Lemma 3.3, $u(s, \varphi)$ is uniformly continuous in $\varphi$ with respect to $s$. Therefore we only need to show that $u(s, \varphi)$ is continuous in $s$ for each $\varphi$.

Let us start by fixing $\varphi \in C$. We desire to know the difference between $u\left(s_{1}, \varphi\right)$ and $u\left(s_{2}, \varphi\right)$ for $0 \leq$ $s_{1} \leq s_{2} \leq T$. From the generalized dynamic programming principle in Theorem 3.7, for all $\epsilon>0$, there exist $v_{1}(\cdot), v_{2}(\cdot) \in \mathcal{U}_{a d}$ such that

$$
G_{s_{1}, s_{2}}^{s_{1}, \varphi ; v_{2}}\left[u\left(s_{2}, X_{s_{2}}^{s_{1}, \varphi ; v_{2}}\right)\right] \leq u\left(s_{1}, \varphi\right) \leq G_{s_{1}, s_{2}}^{s_{1}, \varphi ; v_{1}}\left[u\left(s_{2}, X_{s_{2}}^{s_{1} ; \varphi ; v_{1}}\right)\right]+\epsilon
$$

Then

$$
u\left(s_{1}, \varphi\right)-u\left(s_{2}, \varphi\right) \leq I^{1}+I^{2}+\epsilon
$$

where

$$
\begin{aligned}
I^{1} & =G_{s_{1}, s_{2}}^{s_{1}, v_{1}}\left[u\left(s_{2}, X_{s_{2}}^{s_{1}, \varphi ; v_{1}}\right)\right]-G_{s_{1}, s_{2}}^{s_{1}, \varphi ; v_{1}}\left[u\left(s_{2}, \varphi\right)\right] \\
I^{2} & =G_{s_{1}, s_{2}}^{s_{1}, v_{1}}\left[u\left(s_{2}, \varphi\right)\right]-u\left(s_{2}, \varphi\right) .
\end{aligned}
$$

By the virtue of $G$ (see Refs. Peng [11] or Wu and Yu [12]) and Lemma 3.3, we have

$$
\begin{aligned}
\left|I^{1}\right| & \leq\left(\Lambda \mathbb{E}^{\mathcal{F}_{s_{1}}}\left[\left|u\left(s_{2}, X_{s_{2}}^{s_{1}, \varphi ; v_{1}}\right)-u\left(s_{2}, \varphi\right)\right|^{2}\right]\right)^{\frac{1}{2}} \\
& \leq\left(\Lambda \mathbb{E}^{\mathcal{F}_{s_{1}}}\left[\left\|X_{s_{2}}^{s_{1}, \varphi ; v_{1}}-\varphi\right\|_{L^{2}(\Omega, C)}^{2}\right]\right)^{\frac{1}{2}} .
\end{aligned}
$$

And $I^{2}$ can be rewritten as

$$
\begin{aligned}
I^{2}= & \mathbb{E}^{\mathcal{F}_{s_{1}}}\left[u\left(s_{2}, \varphi\right)+\int_{s_{1}}^{s_{2}} f\left(r, X_{r}^{s_{1}, \varphi ; v_{1}}, Y^{s_{1}, \varphi ; v_{1}}(r), Z^{s_{1}, \varphi ; v_{1}}(r)\right) \mathrm{d} r\right. \\
& \left.+\int_{s_{1}}^{s_{2}} Z^{s_{1}, \varphi ; v_{1}}(r) \mathrm{d} W(r)\right]-u\left(s_{2}, \varphi\right) \\
= & \mathbb{E}^{\mathcal{F}_{s_{1}}}\left[\int_{s_{1}}^{s_{2}} f\left(r, X_{r}^{s_{1}, \varphi ; v_{1}}, Y^{s_{1}, \varphi ; v_{1}}(r), Z^{s_{1}, \varphi ; v_{1}}(r)\right) \mathrm{d} r\right],
\end{aligned}
$$

by the fact that $\mathbb{E}^{\mathcal{F}_{s_{1}}}\left[u\left(s_{2}, \varphi\right)\right]=u\left(s_{2}, \varphi\right)$.

Applying the Schwartz's inequality, and by (A5), Proposition 2.3, we can deduce that

$$
\begin{aligned}
\left|I^{2}\right| & \leq\left(s_{2}-s_{1}\right)^{\frac{1}{2}}\left(\mathbb{E} \int_{s_{1}}^{s_{2}}\left|f\left(r, X_{r}^{s_{1}, \varphi ; v_{1}}, Y^{s_{1}, \varphi ; v_{1}}(r), Z^{s_{1}, \varphi ; v_{1}}(r)\right)\right|^{2} \mathrm{~d} r\right)^{\frac{1}{2}} \\
& \leq C\left(s_{2}-s_{1}\right) .
\end{aligned}
$$

So, letting $s_{2} \downarrow s_{1}$, we can get

$$
u\left(s_{1}, \varphi\right)-u\left(s_{2}, \varphi\right) \leq\left(C \mathbb{E}^{\mathcal{F}_{s_{1}}}\left[\left\|X_{s_{2}}^{s_{1}, \varphi ; v_{1}}-\varphi\right\|_{L^{2}(\Omega, C)}^{2}\right]\right)^{\frac{1}{2}}+C\left(s_{2}-s_{1}\right)+\epsilon \rightarrow 0
$$

as a result of Lemma 4.3 and the arbitrariness of $\epsilon$.

Similarly, we can prove $u\left(s_{2}, \varphi\right)-u\left(s_{1}, \varphi\right) \rightarrow 0$, when $s_{2} \downarrow s_{1}$. Then the continuity of $u$ is proved.

\subsection{HJB equation}

Now we desire to obtain the HJB equation for $u$.

By Lemma 4.1, we have

$$
\mathbb{E}\left[u\left(t+\epsilon, X_{t+\epsilon}\right)-u(t, \varphi)\right]=\int_{t}^{t+\epsilon} \mathbb{E}\left[\frac{\partial}{\partial r} u\left(r, X_{r}\right)+\mathcal{A} u\left(r, X_{r}\right)\right] \mathrm{d} r
$$


i.e.

$$
\mathbb{E}\left[u\left(t, X_{t}^{s, \varphi}\right)\right]=\mathbb{E}\left[u\left(T, X_{T}^{t, X_{t}^{s, \varphi}}\right)\right]-\mathbb{E} \int_{t}^{T}\left[\frac{\partial}{\partial r} u\left(r, X_{r}\right)+\mathcal{A} u\left(r, X_{r}\right)\right] \mathrm{d} r
$$

Since $X_{r}^{t, X_{t}}$ is independent of $\mathcal{F}_{t}$ for $t \leq r \leq T$. Then by the uniqueness of the solution for SDDE, the above equation can be rewritten as

$$
\begin{aligned}
u\left(t, X_{t}^{s, \varphi}\right)= & \mathbb{E}^{\mathcal{F}_{t}}\left[u\left(T, X_{T}^{t, X_{t}^{s, \varphi}}\right)\right]-\mathbb{E}^{\mathcal{F}_{t}} \int_{t}^{T}\left[\frac{\partial}{\partial r} u\left(r, X_{r}\right)+\mathcal{A} u\left(r, X_{r}\right)\right] \mathrm{d} r \\
= & \mathbb{E}^{\mathcal{F}_{t}}\left[u\left(T, X_{T}^{s, \varphi}\right)\right]-\mathbb{E}^{\mathcal{F}_{t}} \int_{t}^{T}\left[\frac{\partial}{\partial r} u\left(r, X_{r}\right)+\mathcal{A} u\left(r, X_{r}\right)\right] \mathrm{d} r \\
= & \mathbb{E}^{\mathcal{F}_{t}}\left[u\left(T, X_{T}^{s, \varphi}\right)\right]-\mathbb{E}^{\mathcal{F}_{t}} \int_{s}^{T}\left[\frac{\partial}{\partial r} u\left(r, X_{r}\right)+\mathcal{A} u\left(r, X_{r}\right)\right] \mathrm{d} r \\
& +\mathbb{E}^{\mathcal{F}_{t}} \int_{s}^{t}\left[\frac{\partial}{\partial r} u\left(r, X_{r}\right)+\mathcal{A} u\left(r, X_{r}\right)\right] \mathrm{d} r \\
= & \mathbb{E}^{\mathcal{F}_{t}}\left[\eta^{*}\right]+\mathbb{E}^{\mathcal{F}_{t}} \int_{s}^{t}\left[\frac{\partial}{\partial r} u\left(r, X_{r}\right)+\mathcal{A} u\left(r, X_{r}\right)\right] \mathrm{d} r
\end{aligned}
$$

where $\eta^{*}=u\left(T, X_{T}^{s, \varphi}\right)-\int_{s}^{T}\left[\frac{\partial}{\partial r} u\left(r, X_{r}\right)+\mathcal{A} u\left(r, X_{r}\right)\right] \mathrm{d} r$. We can see that $\mathbb{E}^{\mathcal{F}_{t}}\left[\eta^{*}\right]$ is a martingale, then by the representation theorem of martingales, there exists a predictable process $\tilde{Z} \in H^{2}$, such that

$$
\begin{aligned}
& \mathbb{E}^{\mathcal{F}_{t}}\left[\eta^{*}\right]=\mathbb{E}^{\mathcal{F}_{s}}\left[\mathbb{E}^{\mathcal{F}_{t}}\left[\eta^{*}\right]\right]+\int_{s}^{t} \tilde{Z}(r) \mathrm{d} W(r) \\
& =u(s, \varphi)+\int_{s}^{t} \tilde{Z}(r) \mathrm{d} W(r),
\end{aligned}
$$

i.e.

$$
u\left(t, X_{t}^{s, \varphi}\right)=u(s, \varphi)+\int_{s}^{t}\left[\frac{\partial}{\partial r} u\left(r, X_{r}^{s, \varphi}\right)+\mathcal{A} u\left(r, X_{r}^{s, \varphi}\right)\right] \mathrm{d} r+\int_{s}^{t} \tilde{Z}(r) \mathrm{d} W(r) .
$$

Then we compute the joint quadratic variation with $W^{i}$ of the processes occurring at both sides of the above equality on an interval $\left[s, T^{\prime}\right] \subset[s, T)$. Considering the right-hand side, we obtain $\int_{s}^{T^{\prime}} \tilde{Z}^{i}(r) \mathrm{d} r$ by the rule of stochastic calculus. By the Theorem 3.7 in [5], we have

$$
\left\langle u\left(\cdot, X^{s, \varphi}\right), W^{i}\right\rangle_{\left[s, T^{\prime}\right]}=\int_{s}^{T^{\prime}} \sigma^{i}\left(r, X_{r}^{s, \varphi}\right) \nabla_{0} u\left(r, X_{r}^{s, \varphi}\right) \mathrm{d} r
$$

Therefore we have $\tilde{Z}(r)=\nabla_{0} u\left(r, X_{r}^{s, \varphi}\right) \sigma\left(r, X_{r}^{s, \varphi}\right)$.

Remark 4.5. Suppose $E=C\left([a, b] ; \mathbb{R}^{n}\right)$, where $a, b \in \mathbb{R}, a<b$. We recall that the dual space of $C([a, b])$ is the space of finite Borel measures on $[a, b]$. Let $u:[0, T] \times C \rightarrow \mathbb{R}$ be a function such that $u(t, \cdot)$ is Gâteaux differentiable on $C$ for every $t \in[0, T]$. Then the gradient $\nabla_{x} u(t, x)$ at point $(t, x) \in[0, T] \times C$ is an n-tuple of finite Borel measures on $[-\delta, 0]$, we denote $\nabla_{x} u(t, x)(\{0\})$ by $\nabla_{0} u(t, x)$.

Then (4.4) can be rewritten as

$$
u\left(t, X_{t}^{s, \varphi}\right)=u(s, \varphi)+\int_{s}^{t}\left[\frac{\partial}{\partial r} u\left(r, X_{r}^{s, \varphi}\right)+\mathcal{A} u\left(r, X_{r}^{s, \varphi}\right)\right] \mathrm{d} r+\int_{s}^{t} \sigma\left(r, X_{r}^{s, \varphi}\right) \nabla_{0} u\left(r, X_{r}^{s, \varphi}\right) \mathrm{d} W(r) .
$$


Also we have

$$
\begin{aligned}
u(s, \varphi)= & u\left(s+\tau, X_{s+\tau}^{s, \varphi}\right)+\int_{s}^{s+\tau}\left[-\frac{\partial}{\partial r} u\left(r, X_{r}^{s, \varphi}\right)-\mathcal{A} u\left(r, X_{r}^{s, \varphi}\right)\right] \mathrm{d} r \\
& -\int_{s}^{s+\tau} \nabla_{0} u\left(r, X_{r}^{s, \varphi}\right) \sigma\left(r, X_{r}^{s, \varphi}\right) \mathrm{d} W(r) .
\end{aligned}
$$

Now let us consider the following BSDE:

$$
Y^{s, \varphi ; v}(s)=u\left(s+\tau, X_{s+\tau}^{s, \varphi}\right)+\int_{s}^{s+\tau} f\left(r, X_{r}^{s, \varphi}, Y^{s, \varphi ; v}(r), Z^{s, \varphi ; v}(r), v(r)\right) \mathrm{d} r-\int_{s}^{s+\tau} Z^{s, \varphi ; v}(r) \mathrm{d} W(r),
$$

and

$$
Y^{1}(s)=\int_{s}^{s+\tau} F\left(r, X_{r}^{s, \varphi}, Y^{1}(r), Z^{1}(r), v(r)\right) \mathrm{d} r-\int_{s}^{s+\tau} Z^{1}(r) \mathrm{d} W(r) .
$$

Here $F(t, x ., y, z, v)=\frac{\partial}{\partial t} u(t, x)+.\mathcal{A} u(t, x)+.f\left(t, x ., y+u(t, x),. z+\nabla_{0} u(t, x.) \sigma, v\right)$.

Proposition 4.6. For any $t \in[s, s+\tau]$, the following relationship holds

$$
Y^{1}(t)=Y^{s, \varphi ; v}(t)-u\left(t, X_{t}^{s, \varphi}\right) .
$$

Proof. Firstly, we have

$$
\begin{aligned}
Y^{s, \varphi ; v}(s)-u(s, \varphi)= & \int_{s}^{s+\tau}\left[f\left(r, X_{r}^{s, \varphi}, Y^{s, \varphi ; v}(r), Z^{s, \varphi ; v}(r), v(r)\right)+\frac{\partial}{\partial r} u\left(r, X_{r}^{s, \varphi}\right)+\mathcal{A} u\left(r, X_{r}^{s, \varphi}\right)\right] \mathrm{d} r \\
& -\int_{s}^{s+\tau}\left[Z^{s, \varphi ; v}(r)-\nabla_{0} u\left(r, X_{r}^{s, \varphi}\right) \sigma\left(r, X_{r}^{s, \varphi}, v(r)\right)\right] \mathrm{d} W(r), \\
Y^{1}(s)= & \int_{s}^{s+\tau}\left[\frac{\partial}{\partial r} u\left(r, X_{r}^{s, \varphi}\right)+\mathcal{A} u\left(r, X_{r}^{s, \varphi}\right)+f\left(r, X_{r}^{s, \varphi}, Y^{1}(r)+u\left(r, X_{r}^{s, \varphi}\right),\right.\right. \\
& \left.\left.Z^{1}(r)+\nabla_{0} u\left(r, X_{r}^{s, \varphi}\right) \sigma\left(r, X_{r}^{s, \varphi}, v(r)\right), v(r)\right)\right] \mathrm{d} r-\int_{s}^{s+\tau} Z^{1}(r) \mathrm{d} W(r) .
\end{aligned}
$$

If we let $Y^{s, \varphi ; v}(\cdot)-u(\cdot, \varphi)=Y^{2}(\cdot), Z^{s, \varphi ; v}(\cdot)-\nabla_{0} u\left(\cdot, X^{s, \varphi}\right) \sigma=Z^{2}(\cdot)$, equation (4.8) can be written as

$$
\begin{aligned}
Y^{2}(s)= & \int_{s}^{s+\tau}\left[\frac{\partial}{\partial r} u\left(r, X_{r}^{s, \varphi}\right)+\mathcal{A} u\left(r, X_{r}^{s, \varphi}\right)+f\left(r, X_{r}^{s, \varphi}, Y^{2}(r)+u\left(r, X_{r}^{s, \varphi}\right),\right.\right. \\
& \left.\left.Z^{2}(r)+\nabla_{0} u\left(r, X_{r}^{s, \varphi}\right) \sigma\left(r, X_{r}^{s, \varphi}, v(r)\right), v(r)\right)\right] \mathrm{d} r-\int_{s}^{s+\tau} Z^{2}(r) \mathrm{d} W(r) .
\end{aligned}
$$

Then by the uniqueness of the solution for BSDE we get

$$
Y^{2}(t)=Y^{1}(t), Z^{2}(t)=Z^{1}(t), t \in[s, s+\tau] \text { a.s. }
$$

Moreover, we can turn to a more simple BSDE than (4.9)

$$
\begin{aligned}
Y^{3}(s)= & \int_{s}^{s+\tau}\left[\frac{\partial}{\partial r} u(r, \varphi)+\mathcal{A} u(r, \varphi)+f\left(r, \varphi, Y^{3}(r)+u(r, \varphi),\right.\right. \\
& \left.\left.Z^{3}(r)+\nabla_{0} u(r, \varphi) \sigma(r, \varphi, v(r)), v(r)\right)\right] \mathrm{d} r-\int_{s}^{s+\tau} Z^{3}(r) \mathrm{d} W(r) .
\end{aligned}
$$

Then we can derive the following estimate about the solutions of (4.9) and (4.11). 
Lemma 4.7. For BSDEs (4.9) and (4.11), we have

$$
\left|Y^{1}(s)-Y^{3}(s)\right| \leq \Lambda \tau \rho_{1}(\tau)
$$

where $\rho_{1}(\cdot): \mathbb{R}^{+} \rightarrow \mathbb{R}$ is a function such that $\rho_{1}(\tau) \downarrow 0$ when $\tau \downarrow 0$ and $\rho_{1}(\cdot)$ is independent of the control $v(\cdot)$.

Proof. From Proposition 2.1, we have

$$
\left\|X_{t}^{s, \varphi ; v}\right\|_{L^{2}(\Omega, C)}^{2}=\mathbb{E}^{\mathcal{F}_{s}}\left[\left\|X_{t}^{s, \varphi ; v}\right\|_{C}^{2}\right] \leq \Lambda\left(1+\|\varphi\|_{C}^{2}\right) .
$$

And from Lemma 4.3, we have

$$
\left\|X_{t}^{s, \varphi ; v}-\varphi\right\|_{L^{2}(\Omega, C)}^{2} \rightarrow 0, \text { when } t \rightarrow s^{+} .
$$

For BSDEs (4.9) and (4.11), we have the following estimates

$$
\begin{aligned}
& \mathbb{E}^{\mathcal{F}_{s}} \int_{s}^{s+\tau}\left[\left|Y^{1}(t)-Y^{3}(t)\right|^{2}+\left|Z^{1}(s)-Z^{3}(t)\right|^{2}\right] \mathrm{d} t \\
\leq & \Lambda \mathbb{E}^{\mathcal{F}_{s}} \int_{s}^{s+\tau}\left[\rho^{2}\left(\left\|X_{t}^{s, \varphi ; v}-\varphi\right\|_{C}\right)\right]^{2} \mathrm{~d} t \\
\leq & \Lambda \tau \mathbb{E}^{\mathcal{F}_{s}} \rho^{2}\left(\beta^{\tau}\right),
\end{aligned}
$$

where $\rho(\cdot)$ satisfies $\rho(\epsilon) \rightarrow 0$ when $\epsilon \rightarrow 0$ and $\rho(\epsilon) \leq C(1+\epsilon)$ for all $\epsilon \geq 0$. And $\beta^{\tau}=\sup _{t \in[s, s+\tau]}\left\|X_{t}^{s, \varphi ; v}-\varphi\right\|_{C}$. By Lemma 4.3, we have $\beta^{\tau} \downarrow 0$ when $t \downarrow s^{+}$.

On the other hand, since $Y^{1}(s)$ and $Y^{3}(s)$ are $\mathcal{F}_{s}$-measurable, then

$$
\begin{aligned}
& \left|Y^{1}(s)-Y^{3}(s)\right|=\left|\mathbb{E}^{\mathcal{F}_{s}}\left(Y^{1}(s)-Y^{3}(s)\right)\right| \\
= & \left|\mathbb{E}^{\mathcal{F}_{s}} \int_{s}^{s+\tau}\left[F\left(t, X_{t}^{s, \varphi ; v}, Y^{1}(t), Z^{1}(t), v(t)\right)-F\left(t, \varphi, Y^{3}(t), Z^{3}(t), v(t)\right)\right] \mathrm{d} t\right| \\
\leq & \mathbb{E}^{\mathcal{F}_{s}} \int_{s}^{s+\tau}\left[\rho\left(\left\|X_{t}^{s, \varphi ; v}-\varphi\right\|_{C}\right)+\Lambda\left|Y^{1}(t)-Y^{3}(t)\right|+\Lambda\left|Z^{1}(t)-Z^{3}(t)\right|\right] \mathrm{d} t \\
\leq & \tau \mathbb{E}^{\mathcal{F}_{s}} \rho\left(\beta^{\tau}\right)+\Lambda \tau^{\frac{1}{2}}\left\{\mathbb{E}^{\mathcal{F}_{s}} \int_{s}^{s+\tau}\left(\left|Y^{1}(t)-Y^{2}(t)\right|^{2}+\left|Z^{1}(t)-Z^{2}(t)\right|^{2}\right) \mathrm{d} t\right\}^{\frac{1}{2}}
\end{aligned}
$$

So

$$
\left|Y^{1}(s)-Y^{3}(s)\right| \leq \Lambda \tau\left\{\mathbb{E}^{\mathcal{F}_{s}}\left[\rho\left(\beta^{\tau}\right)\right]+\left\{\mathbb{E}^{\mathcal{F}_{s}}\left[\rho^{2}\left(\beta^{\tau}\right)\right]\right\}^{\frac{1}{2}}\right\} .
$$

Notice that $\rho\left(\beta^{\tau}\right)$ is square-integrable for each $\tau>0$, then we have

$$
\left|Y^{1}(s)-Y^{3}(s)\right| \leq \Lambda \tau \rho_{1}(\tau) .
$$

Lemma 4.8. We have

$$
\sup _{v(\cdot) \in \mathcal{U}_{a d}} Y^{3}(s)=Y^{0}(s)
$$

where $Y^{0}(\cdot)$ is the solution of the following ordinary differential equation

$$
\left\{\begin{array}{l}
-\dot{Y}^{0}(t)=F_{0}\left(t, \varphi, Y^{0}(t), 0\right), t \in[s, s+\tau], \\
Y^{0}(s+\tau)=0,
\end{array}\right.
$$

here $F_{0}(t, \varphi, y, z)=\sup _{v \in U} F(t, \varphi, y, z, v)$. 
Proof. By the definition of $F_{0}$, we know

$$
F_{0}(t, \varphi, y, z) \geq F(t, \varphi, y, z, v(t)), \forall v(\cdot) \in \mathcal{U}_{a d}, \forall \varphi, y, z, t .
$$

From the comparison theorem of BSDE, we have

$$
Y^{3}(t) \leq Y^{0}(t), t \in[s, s+\tau], \forall v(\cdot) \in \mathcal{U}_{a d},
$$

where $\left(Y^{0}, Z^{0}\right)$ is the solution of the following BSDE

$$
\left\{\begin{aligned}
-\mathrm{d} Y^{0}(t) & =F_{0}\left(t, \varphi, Y^{0}(t), Z^{0}(t)\right) \mathrm{d} t-Z^{0}(t) \mathrm{d} W(t), t \in[s, s+\tau], \\
Y^{0}(s+\tau) & =0 .
\end{aligned}\right.
$$

Noticing that $F_{0}$ is a deterministic function, we have the solution of $(4.12)$ is $\left(Y^{0}(t), 0\right)$, where $Y^{0}(t)$ satisfies

$$
\left\{\begin{array}{l}
-\dot{Y}^{0}(t)=F_{0}\left(t, \varphi, Y^{0}(t), 0\right), t \in[s, s+\tau] \\
Y^{0}(s+\tau)=0
\end{array}\right.
$$

By the above analysis, we know that, if we can prove

$$
Y^{0}(s)=\sup _{v(\cdot) \in \mathcal{U}_{0}} Y^{3}(s)
$$

$\mathcal{U}_{0}$ is the collection of deterministic admissible controls, then the desired conclusion can be obtained. In fact, for each $v(\cdot) \in \mathcal{U}_{0}, Y^{3}(t)$ is the solution of

$$
\left\{\begin{array}{l}
-\dot{Y}^{3}(t)=F\left(t, \varphi, Y^{3}(t), 0, v(t)\right), t \in[s, s+\tau], \\
Y^{3}(s+\tau)=0 .
\end{array}\right.
$$

So our lemma can be obtained obviously.

Now we can obtain our HJB equations from the dynamic programming principle for the delayed system.

Theorem 4.9. If we assume that the value function $u(s, \varphi) \in C_{\text {lip }}^{1,2}([0, T] \times C) \cap D(\mathcal{S})$ in our stochastic recursive optimal control problem, then $u(s, \varphi)$ solves the following Hamilton-Jacobi-Bellman partial differential equation

$$
\frac{\partial}{\partial s} u(s, \varphi)+\sup _{v \in U}\left\{\mathcal{A} u(s, \varphi)+f\left(s, \varphi, u(s, \varphi), \nabla_{0} u(s, \varphi) \sigma, v\right)\right\}=0,
$$

with the terminal condition $u(T, \varphi)=\Phi(\varphi)$.

Proof. From the general dynamic programming principle-Theorem 3.7, we have

$$
\begin{aligned}
u(s, \varphi) & =\sup _{v(\cdot) \in \mathcal{U}_{a d}} G_{s, s+\tau}^{s, \varphi}\left[u\left(s+\tau, X_{s+\tau}^{s, \varphi ; v}\right)\right] \\
& =\sup _{v(\cdot) \in \mathcal{U}_{a d}} Y^{s, \varphi ; v}(s) .
\end{aligned}
$$

So we can get $Y^{2}(s) \leq 0$ in $(4.10)$ and $\sup _{v(\cdot) \in \mathcal{U}_{a d}} Y^{2}(s) \leq 0$, i.e.

$$
\sup _{v(\cdot) \in \mathcal{U}_{a d}} \int_{s}^{s+\tau} F\left(t, X_{t}^{s, \varphi ; v}, Y^{2}(t), Z^{2}(t), v(t)\right) \mathrm{d} t \leq 0 .
$$


By the fact that $F$ is Lipsichtz with respect to $(\varphi, y, z)$ and Lemma 4.7, we have

$$
\begin{aligned}
\mathbb{E} \int_{s}^{s+\tau} F\left(r, \varphi, Y^{3}(r), Z^{3}(r), v(r)\right) \mathrm{d} r-\rho_{1}(\tau) & \leq \mathbb{E} \int_{s}^{s+\tau} F\left(r, X^{s, \varphi ; v}(r), Y^{2}(r), Z^{2}(r), v(r)\right) \mathrm{d} r \\
& \leq 0 .
\end{aligned}
$$

This will lead to

$$
\sup _{v(\cdot) \in \mathcal{U}_{a d}} Y^{3}(s) \leq \rho_{1}(\tau)
$$

From Lemma 4.8, we have

$$
Y^{0}(s) \leq 0, \tau \downarrow 0
$$

and

$$
F_{0}(s, \varphi, 0,0)=\sup _{v \in U} F(s, \varphi, 0,0, v) \leq 0 .
$$

On the other hand, there exists a $v(\cdot)$ s.t. for a small enough $\tau$,

$$
u(s, \varphi) \leq Y^{3}(s)+\epsilon \tau,
$$

i.e.

$$
Y^{2}(s) \geq-\epsilon \tau
$$

That implies

$$
\begin{aligned}
& \sup _{v(\cdot) \in \mathcal{U}_{a d}} \mathbb{E} \int_{s}^{s+\tau} F\left(r, \varphi, Y^{3}(r), Z^{3}(r), v(r)\right) \mathrm{d} r \geq-\epsilon \tau-C \rho_{1}(\tau), \\
& \sup _{v(\cdot) \in \mathcal{U}_{a d}} Y^{3}(s) \geq 0,
\end{aligned}
$$

and $F_{0}(s, \varphi, 0,0)=\sup _{v \in U} F(s, \varphi, 0,0, v) \geq 0$.

Then

$$
\sup _{v \in U} F(s, \varphi, 0,0, v)=0
$$

i.e.

$$
\frac{\partial}{\partial s} u(s, \varphi)+\sup _{v \in U}\left\{\mathcal{A} u(s, \varphi)+f\left(s, \varphi, u(s, \varphi), \nabla_{0} u(s, \varphi) \sigma, v\right)\right\}=0 .
$$

\subsection{Viscosity solution of the HJB equation}

In this section, we will show that the value function $u$ defined by (3.1) is actually a viscosity solution of the HJB equation (4.13). Firstly, let us define the viscosity solution of (4.13) as follows.

Definition 4.10. Let $\Psi \in C([0, T] \times C)$.

(a) We say that $\Psi$ is a viscosity subsolution of (4.13) if

$$
\Psi(T, \varphi) \leq \Phi(\varphi), \text { for all } \varphi \in C
$$

and for any $\Gamma \in C_{\text {lip }}^{1,2}([0, T] \times C) \cap D(\mathcal{S})$, whenever $\Gamma-\Psi$ attains a local minimum at $(s, \varphi)$, we have

$$
\frac{\partial}{\partial s} \Gamma(s, \varphi)+\sup _{v \in U}\left\{\mathcal{A} \Gamma(s, \varphi)+f\left(s, \varphi, \Gamma(s, \varphi), \nabla_{0} \Gamma(s, \varphi) \sigma, v\right)\right\} \geq 0 .
$$


(b) We say that $\Psi$ is a viscosity supersolution of (4.13) if

$$
\Psi(T, \varphi) \geq \Phi(\varphi), \text { for all } \varphi \in C
$$

and for any $\Gamma \in C_{\text {lip }}^{1,2}([0, T] \times C) \cap D(\mathcal{S})$, whenever $\Gamma-\Psi$ attains a local maximum at $(s, \varphi)$, we have

$$
\frac{\partial}{\partial s} \Gamma(s, \varphi)+\sup _{v \in U}\left\{\mathcal{A} \Gamma(s, \varphi)+f\left(s, \varphi, \Gamma(s, \varphi), \nabla_{0} \Gamma(s, \varphi) \sigma, v\right)\right\} \leq 0 .
$$

(c) $\Psi \in C([0, T] \times C)$ is said to be a viscosity solution of (4.13) if it is both a viscosity sub- and supersolution.

Theorem 4.11. $u(s, \varphi)$ defined by (3.1) is a viscosity solution of HJB equation (4.13).

Proof. The proof of Theorem 4.11 is similar to the Theorem 4.9. We will outline the proof.

Let us define

$$
H(s, \varphi, y, z, v)=\frac{\partial \Gamma(s, \varphi)}{\partial s}+\mathcal{A} \Gamma(s, \varphi)+f\left(s, \varphi, y+\Gamma(s, \varphi), z+\nabla_{0} \Gamma(s, \varphi) \sigma, v\right) .
$$

We also consider the following BSDE defined on $[s, s+\tau]$,

$$
\left\{\begin{array}{l}
-\mathrm{d} Y^{4}(t)=H\left(t, X_{t}^{s, \varphi ; v}, Y^{4}(t), Z^{4}(t), v(t)\right) \mathrm{d} t \\
-Z^{4}(t) \mathrm{d} W(t), t \in[s, s+\tau] \\
Y^{4}(s+\tau)=0
\end{array}\right.
$$

Same as Proposition 4.6, we have the following relationship holds

$$
Y^{4}(t)=G_{t, s+\tau}^{s, \varphi ; v}\left[\Gamma\left(s+\tau, X_{s+\tau}^{s, \varphi ; v}\right)\right]-\Gamma\left(t, X_{t}^{s, \varphi ; v}\right), \text { a.s. },
$$

for any $t \in[s, s+\tau]$. We also have

$$
\left|Y^{4}(s)-Y^{5}(s)\right| \leq C \tau \rho_{1}(\tau),
$$

here $\rho_{1}(\tau)$ is the same as Lemma 4.7 , and

$$
\left\{\begin{array}{l}
-\mathrm{d} Y^{5}(t)=H\left(t, \varphi, Y^{5}(t), Z^{5}(t), v(t)\right) \mathrm{d} t-Z^{5}(t) \mathrm{d} W(t), t \in[s, s+\tau] \\
Y^{5}(s+\tau)=0
\end{array}\right.
$$

with $\varphi \in C$.

We can also prove that

$$
\sup _{v(\cdot) \in \mathcal{U}_{a d}} Y^{5}(s)=Y^{6}(s)
$$

where

$$
\left\{\begin{array}{l}
-\dot{Y}^{6}(t)=H_{0}\left(t, \varphi, Y^{6}(t), 0\right), t \in[s, s+\tau] \\
Y^{6}(s+\tau)=0
\end{array}\right.
$$

with $H_{0}(s, \varphi, y, z) \equiv \sup _{v \in U} H(s, \varphi, y, z, v)$.

Let $\Gamma \in C_{l i p}^{1,2}([0, T] \times C) \cap D(\mathcal{S})$ and $\Gamma-u$ attains minimum (maximum) at $(s, \varphi)$, and $\Gamma(s, \varphi)=u(s, \varphi)$. Then from the dynamic programming principle-Theorem 3.7 , we have

$$
\Gamma(s, \varphi)=u(s, \varphi)=\sup _{v(\cdot) \in \mathcal{U}_{a d}} G_{s, s+\tau}^{s, \varphi ; v}\left[u\left(s+\tau, X_{s+\tau}^{s, \varphi ; v}\right)\right]
$$


From $\Gamma \geq u$ (resp., $\Gamma \leq u$ ) and the monotone of $G$ we have

$$
\sup _{v(\cdot) \in \mathcal{U}_{a d}}\left\{G_{s, s+\tau}^{s, \varphi ; v}\left[u\left(s+\tau, X_{s+\tau}^{s, \varphi ; v}\right)\right]-\Gamma(s, \varphi)\right\} \geq 0,(\text { resp. }, \leq 0) .
$$

From (4.14), we can get

$$
\sup _{v(\cdot) \in \mathcal{U}_{a d}} Y^{4}(s) \geq 0(\text { resp. }, \leq 0)
$$

Then

$$
\sup _{v(\cdot) \in \mathcal{U}_{a d}} Y^{5}(s) \geq-\tau \rho_{1}(\tau)\left(\text { resp. }, \leq \tau \rho_{1}(\tau)\right)
$$

Moreover, by (4.16),

$$
Y^{6}(s) \geq-\tau \rho_{1}(\tau)\left(\text { resp. }, \leq \tau \rho_{1}(\tau)\right)
$$

Consequently,

$$
H_{0}(s, \varphi, 0,0)=\sup _{v \in U} H(s, \varphi, 0,0, v) \geq 0(\text { resp. }, \leq 0) .
$$

Then from the definition of $H$ we know that $u$ is a viscosity subsolution (resp., supersolution) of HJB equation (4.13). Thus $u$ is the viscosity solution of (4.13).

Remark 4.12. The uniqueness for the viscosity solution of equation (4.13) is not easy to be obtained. However, for some special case, we can prove that the value function defined by (3.1) is the unique viscosity solution of the corresponding HJB equation.

We consider the controlled delayed system (2.2) with coefficients satisfying (A1)-(A3). The cost functional is given by $J(s, \varphi ; v(\cdot))=\left.Y(t)\right|_{t=s},(s, \varphi) \in[0, T] \times C$, where $Y$ is a solution of the BSDE

$$
\left\{\begin{array}{l}
-\mathrm{d} Y(t)=\left[a\left(t, X_{t}, v(t)\right)+b(t) Y(t)+g(t) Z(t)\right] \mathrm{d} t-Z(t) \mathrm{d} W(t), \\
Y(T)=\Phi\left(X_{T}\right) .
\end{array}\right.
$$

We also need the following assumptions:

(A6) $a(\cdot, \cdot, \cdot):[0, T] \times C \times U \rightarrow \mathbb{R}$ and $\Phi(\cdot): C \rightarrow \mathbb{R}$ satisfy (A4)-(A5). Moreover, $b(\cdot), g(\cdot)$ are given uniformly bounded deterministic functions with respect to $t \in[0, T]$.

Then, by the Girsanov's theorem, we can rewrite (2.2) and (4.17) as

$$
\left\{\begin{array}{l}
\mathrm{d} X(t)=\left[b\left(t, X_{t}, v(t)\right)+\sigma\left(t, X_{t}, v(t)\right) g(t)\right] \mathrm{d} t+\sigma\left(t, X_{t}, v(t)\right) d \bar{W}(t), t \in[s, T], \\
X_{s}=\varphi_{s} \\
-\mathrm{d} Y(t)=\left[a\left(t, X_{t}, v(t)\right)+b(t) Y(t)\right] \mathrm{d} t-Z(t) d \bar{W}(t) \\
Y(T)=\Phi\left(X_{T}\right) .
\end{array}\right.
$$

Here $\bar{W}(t)=W(t)-\int_{0}^{t} g(s) \mathrm{d} s$. We define a probability measure $Q$ on $(\Omega, \mathcal{F})$ by

$$
\frac{d Q}{d P}=\exp \left\{\int_{0}^{T} g(t) \mathrm{d} W(t)-\frac{1}{2} \int_{0}^{T} g^{2}(t) \mathrm{d} t\right\} .
$$

Obviously, $\bar{W}(t)$ is a standard Brownian motion on $(\Omega, \mathcal{F}, Q)$. By the definition of $\mathcal{U}_{\text {ad }}$, we known that there exists a unique solution of (4.18).

Under the new probability measure $Q$, the cost functional can be written as

$$
J(s, \varphi ; v(\cdot))=\mathbb{E}_{Q}\left[\int_{s}^{T} \mathrm{e}^{\int_{s}^{t} b(r) \mathrm{d} r} a\left(t, X_{t}, v(t)\right) \mathrm{d} t+\mathrm{e}^{\int_{s}^{T} b(r) \mathrm{d} r} \Phi\left(X_{T}\right)\right] .
$$


Then we have the following result:

Proposition 4.13. We define the value function of the above recursive optimal control problem with delay by $u(s, \varphi)=\operatorname{esssup}_{v(\cdot) \in \mathcal{U}_{a d}} J(s, \varphi ; v(\cdot)),(s, \varphi) \in[0, T] \times C$. Then $u(s, \varphi)$ is a viscosity solution of the following equation

$$
\frac{\partial}{\partial s} u(s, \varphi)+\sup _{v \in U}\{\mathcal{A} u(s, \varphi)+a(s, \varphi, v)\}=0
$$

with $u(T, \varphi)=\mathrm{e}^{\int_{s}^{T} b(r) \mathrm{d} r} \Phi(\varphi)$. Moreover, there exists unique viscosity solution of HJB equation (4.19) in the class of continuous functions with at most a polynomially growth.

Proof. First, by Theorem 4.11, we know that the value function $u(s, \varphi)$ is a viscosity solution of (4.19). And from Lemma 3.3 and Proposition 4.4 we can see that equation (4.19) has at least one solution in the class of continuous functions with at most a polynomial growth.

On the other hand, if $u^{1}(s, \varphi)$ and $u^{2}(s, \varphi)$ are both continuous viscosity solutions of (4.19) which grow at most polynomially, i.e. there exists a constant $\Lambda>0$ and a positive integer $k>0$ such that

$$
\left|u^{i}(s, \varphi)\right| \leq \Lambda\left(1+\|\varphi\|_{2}\right)^{k},(s, \varphi) \in[0, T] \times C, i=1,2 .
$$

By the definition of viscosity solution, without loss of generality, we can suppose $u^{1}(s, \varphi)$ and $u^{2}(s, \varphi)$ are respectively viscosity subsolution and supersolution of (4.19). Then applying the comparison principle in Theorem 5.1 of [1], we have

$$
u^{1}(s, \varphi) \leq u^{2}(s, \varphi),(s, \varphi) \in[0, T] \times C .
$$

Conversely, we also have

$$
u^{1}(s, \varphi) \geq u^{2}(s, \varphi),(s, \varphi) \in[0, T] \times C .
$$

We have $u^{1}(s, \varphi)=u^{2}(s, \varphi)$ immediately. The proof is completed.

Acknowledgements. The authors are particularly grateful to Dr. Zhiyong Yu for his constructive suggestions and discussion related to this work.

\section{REFERENCES}

[1] M. Chang, T. Pang and M. Pemy, Optimal control of stochastic functional differential equations with a bounded memory. Stochastic An International J. Probability \& Stochastic Process 80 (2008) 69-96.

[2] D. Duffie and L.G. Epstein, Stochastic differential utility. Economicrica 60 (1992) 353-394.

[3] N. El Karoui, S. Peng and M.C. Quenez, Backward stochastic differential equation in finance. Math. Finance 7 (1997) 1-71.

[4] M. Fuhrman and G. Tessitore, Nonlinear Kolmogorov equation in infinite dimensional spaces: the backward stochastic differential equations approach and applications to optimal control. Ann. Probab. 30 (2002) 1397-1465.

[5] M. Fuhrman, F. Masiero and G. Tessitore, Stochastic equations with delay: optimal control via BSDEs and regular solutions of Hamilton-Jacobi-Bellman equations. SIAM J. Control Optim. 48 (2010) 4624-4651.

[6] B. Larssen, Dynamic programming in stochastic control of systems with delay. Stoch. Stoch. Rep. 74 (2002) 651-673.

[7] B. Larssen and N.H. Risebro, When are HJB equations for control problems with stochastic delay equations finite dimensional? Dr. Scient. thesis, University of Oslo (2003).

[8] S.E.A. Mohammed, Stochastic Functional Differential Equations, Pitman (1984).

[9] S.E.A. Mohammed, Stochastic Differential Equations with Memory: Theory, Examples and Applications, Stochastic Analysis and Related Topics 6. The Geido Workshop (1996); Progress in Probability. Birkhauser (1998).

[10] S. Peng, A generalized dynamic programming principle and Hamilton-Jacobi-Bellmen equation. Stoch. Stoch. Rep. 38 (1992) 119-134.

[11] S. Peng, Backward stochastic differential equations-stochastic optimization theory and viscosity solution of HJB equations. Topics on Stochastic Analysis (in Chinese), edited by J. Yan, S. Peng, S. Fang and L. Wu. Science Press, Beijing (1997) 85-138.

$[12] \mathrm{Z}$. Wu and Z. Yu, Dynamic programming principle for one kind of stochastic recursive optimal control problem and HamiltonJacobi-Bellman equation. SIAM J. Control Optim. 47 (2008) 2616-2641.

[13] J. Yong and X.Y. Zhou, Stochastic Controls. Springer-Verlag (1999). 